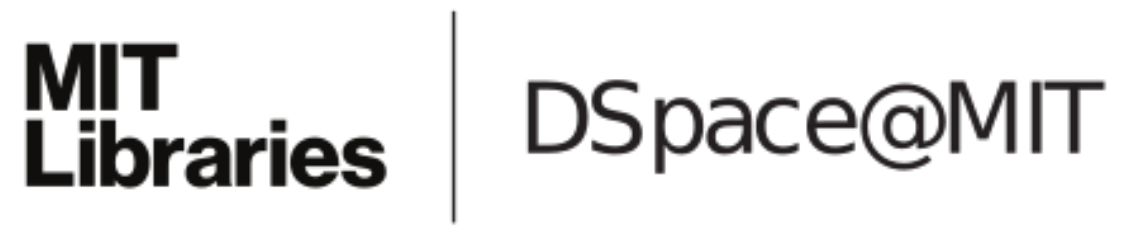

\author{
MIT Open Access Articles
}

Randomness and Nonlinear Evolution Equations

The MIT Faculty has made this article openly available. Please share how this access benefits you. Your story matters.

Citation: Nahmod, Andrea R. and Gigliola Staffilani. "Randomness and Nonlinear Evolution Equations." Acta Mathematica Sinica, English Series 35, 6 (June 2019): 903-932 ( 2019 Springer Nature Switzerland AG

As Published: http://dx.doi.org/10.1007/S10114-019-8297-5

Publisher: Springer Science and Business Media LLC

Persistent URL: https://hdl.handle.net/1721.1/125076

Version: Author's final manuscript: final author's manuscript post peer review, without publisher's formatting or copy editing

Terms of use: Creative Commons Attribution-Noncommercial-Share Alike 
Acta Mathematica Sinica, English Series

Sep., 201x, Vol. x, No. x, pp. 1-30

Published online: August 15, 201x

DOI: 0000000000000000

Http://www.ActaMath.com

\title{
Randomness and nonlinear evolution equations
}

\author{
Andrea R. NAHMOD \\ Department of Mathematics, University of Massachusetts Amherst, 710 N. Pleasant Street, Amherst \\ MA 01003 \\ nahmod@math.umass.edu \\ Gigliola STAFFILANI \\ Department of Mathematics, Massachusetts Institute of Technology, 77 Massachusetts Avenue, \\ Cambridge, MA 02139 \\ gigliola@math.mit.edu
}

\begin{abstract}
In this paper we survey some results on existence, and when possible also uniqueness, of solutions to certain evolution equations obtained by injecting randomness either on the set of initial data or as a perturbative term.
\end{abstract}

Keywords Probabilistic well-posedness, Gibbs measure, invariance.

MR(2010) Subject Classification 35A05, 35B10, 35E15, 35Q35, 35Q55, 35R60

\section{Introduction}

In the last twenty five years the field of dispersive PDE has seen incredible advances, from the well-posedness theory to the blow up analysis, from the study of soliton interactions to partial results on the "soliton resolution conjecture". The contribution of Carlos Kenig in this large body of collective work is staggering. Let us just mention some of the most fundamental results that he and collaborators produced: [3, 4, 21-23, 26-28, 37-41, 43, 44, 46-50].

In this survey paper we take a different approach, we focus on well-posedness type results that are generic in nature, more than deterministically obtained. In fact, since the pioneering work of Bourgain on almost sure (a.s.) global well-posedness of certain dispersive equations via the invariance of the Gibbs measure [8,9], a large body of work has been produced based on the effectiveness of initial data randomization to obtain theorems on existence (and sometimes uniqueness) of solutions for certain evolution equations on low regime of regularity, a situation in which deterministic tools often may no longer be suitable. For the purpose of this survey we can group these theorems in three distinct classes. The the first class collects results in which a local well-posedness is extended to a global one by using the invariance, or sometime almost invariance, of a Gibbs type measure. This includes for example [8], [9] and the example that will be recalled below and studied in [54, 57], see Section 2 and 3. The second class, see Section 4, contains problems in which local well-posedness or existence of weak solutions

Received x x, 201x, accepted x x, 201x

The first author was supported by NSF (DMS 1201443 and DMS 1463714), the second author by NSF (DMS 1362509 and DMS 1462401), the Simons Foundation and the John Simon Guggenheim Foundation. 
can be obtained at a very low level of regularity by studying the so called difference equation. The solution $w$ of the difference equation is such that $w=u-W(t) u_{0}^{\omega}$, where $u$ and $W(t)$ are respectively the solution of the original equation and its linear operator, and $u_{0}^{\omega}$ is the random initial data. The fundamental fact used here is that the initial value problem satisfied by $w$ is more regular than the one originally given for $u$ and hence deterministic techniques can be used. In this class we can include, besides [8, 9] already recalled above, the works $[14,15]$ where the problem is set on a compact manifolds, $[12,24]$ where the equation has a discrete spectrum thanks to the presence of a potential, and $[5,6,25,52,53]$ where the ambient space is $\mathbb{R}^{d}$ and the randomization is obtained by reassembling the Fourier transform of the initial data by randomly summing a partition of unity supported on unit cubes, in other words, randomizing a Wiener decomposition of the initial data. In this class we will survey in particular [58] for the quintic periodic 3D NLS, and [55] for the periodic 2D and 3D Navier-Stokes equations. The third class is concerned with a procedure originally introduced for certain fluid equations by Albeverio and Cruzeiro [1], see Section 5. In this procedure an invariant measure is associated to the equation at hand and one shows that on the support of the measure the expectation of the nonlinear part of the truncated equation is finite uniformly with respect to the truncation parameter. Starting from here a Galerkin approximation scheme is then set up and a probabilistic convergence argument is presented via the Prokorov's Lemma (for compactness) and the Skorokhod's Lemma (for convergence of measures). Unfortunately in this procedure uniqueness of solutions is often difficult to achieve, even in a more probabilistic sense; see e.g. [1], [13], [59], [56]. Below, in Section 5 we survey our results in [56]. In the same section we survey the work in [11], thus giving an example of regularization by noise (c.f. [30]) in the context of the inviscid quasi-geostrophic equation where randomness is injected as a perturbative term in the equation itself.

Before we pass to specific equations and problems we give a precise definition of what we mean for randomizing initial data. Here we assume that the base space is $H^{s}\left(\mathbb{T}^{d}\right)$ for $s \in \mathbb{R}$, but this procedure can be applied also for spaces of Lebesgue type such as the ones used in [54] for example.

Definition 1.1 Let $\left(g_{n}(\omega)\right)_{n \in \mathbb{Z}^{d}}$ be a sequence of complex i.i.d centered Gaussian random variables on a probability space $(\Omega, A, P)$. For $\phi \in H^{s}\left(\mathbb{T}^{3}\right)$, let $\left(b_{n}\right)$ be its Fourier coefficients, that is

$$
\phi(x)=\sum_{n \in \mathbb{Z}^{d}} b_{n} e^{i n \cdot x}, \quad \sum_{n \in \mathbb{Z}^{d}}(1+|n|)^{2 s}\left|b_{n}\right|^{2}<\infty .
$$

The map from $(\Omega, A)$ to $H^{s}\left(\mathbb{T}^{d}\right)$ equipped with the Borel sigma algebra, defined by

$$
\omega \longrightarrow \phi^{\omega}, \quad \phi^{\omega}(x)=\sum_{n \in \mathbb{Z}^{d}} g_{n}(\omega) b_{n} e^{i n \cdot x}
$$

is called a map randomization.

Remark 1.2 The map (1.2) is measurable and $\phi^{\omega} \in L^{2}\left(\Omega ; H^{s}\left(\mathbb{T}^{d}\right)\right)$, is an $H^{s}\left(\mathbb{T}^{d}\right)$-valued random variable. We remark again that such a randomization does not introduce any $H^{s}$ regularization (see Lemma B.1 in [14] for a proof of this fact), indeed $\left\|\phi^{\omega}\right\|_{H^{s}} \sim\|\phi\|_{H^{s}}$. However randomization gives improved $L^{p}$ estimates almost surely. 
We also observe that for any set $\tilde{\Omega} \subset \Omega$ and any $\phi \in H^{s}$, we can define sets $\Sigma \subset H^{s}$ such that

$$
\Sigma:=\left\{\phi^{\omega} / \omega \in \tilde{\Omega}\right\}
$$

and a measure $\mu$ such that

$$
\mu(\Sigma):=P(\tilde{\Omega})
$$

\section{Almost Sure global well-posedness and (almost) invariance of the Gibbs measure}

The general strategy for problems in this class is to start with a Gibbs measure defined on a set of (rough) initial data. Then one first proves, either deterministically [8] or probabilistically $[9,14]$, that there is a local flow on this set of data, and then the flow is extended for all times using the invariance of the measure. Of course this argument needs first to be implemented on the truncated initial value problem, and then a limit process is activated.

In this section we consider the 1D periodic derivative non linear Schrödinger DNLS equation equation studied in [54]. For this example the general argument outlined above does not work as stated due to the fact that a gauge transformation needs to be implemented in order to be able to show that a local flow is available.

We now give some details of how the argument needs to be refined in order to obtain almost sure global well-posedness for the DNLS, and we refer the reader to the original paper [54] for a full history of the problem and a complete bibliography.

The periodic initial value problem for DNLS takes the form:

$$
\left\{\begin{array}{l}
u_{t}-i u_{x x}=\lambda\left(|u|^{2} u\right)_{x} \\
\left.u\right|_{t=0}=u_{0},
\end{array}\right.
$$

where $(x, t) \in \mathbb{T} \times(-T, T)$ and $\lambda$ is real. In this paper we will take $\lambda=1$ for convenience. DNLS is a Hamiltonian PDE whose flow conserves also mass and energy; i.e. the following are conserved quantities of time ${ }^{1}$ :

$$
\begin{aligned}
\text { Mass: } \quad m(u)(t) & =\int|u(x, t)|^{2} d x, \\
\text { Energy: } & E(u)(t)=\int\left|u_{x}\right|^{2} d x+\frac{3}{2} \operatorname{Im} \int u^{2} \bar{u} \overline{u_{x}} d x+\frac{1}{2} \int|u|^{6} d x, \\
\text { Hamiltonian: } & H(u)(t)=\operatorname{Im} \int u \bar{u}_{x} d x+\frac{1}{2} \int|u|^{4} d x .
\end{aligned}
$$

The original motivation for the work in [54] was to prove the invariance of the Gibbs measure defined in [63] using the mass and the energy defined above. Informally this measure can be written as

$$
\text { " } d \nu=\frac{1}{\tilde{Z}} e^{-E(u)-m(u)} d u ",
$$

where $Z$ is a normalizing constant and $E$ and $M$ are defined above. But to make sense of this measure one needs to use the Gaussian measure [7]

$$
d \rho:=\frac{1}{Z} e^{-\int_{\mathbb{T}}\left|u_{x}\right|^{2} d x-m(u)} d u
$$

\footnotetext{
${ }^{1}$ In fact, DNLS is completely integrable.
} 
supported in spaces that scale like $H^{s}(\mathbb{T}), s<\frac{1}{2}$, and show that the remaining part of $d \mu$, namely

$$
e^{-\frac{3}{2} \operatorname{Im} \int u^{2} \bar{u} \overline{u_{x}} d x-\frac{1}{2} \int|u|^{6} d x}
$$

that contains the nonlinear terms of $E(u)$, is in $L^{1}$ with respect to $d \rho$.

Although in [63] the Gibbs measure was defined, the first step in order to prove invariance is to show that the equation itself has a well defined global in time flow in the support of the measure. Unfortunately the well-posedness question for the initial value problem (2.1) is made complicated by a derivative loss coming from the nonlinearity $\left(|u|^{2} u\right)_{x}=u^{2} \bar{u}_{x}+2|u|^{2} u_{x}$, more precisely from the second term of this sum. To resolve this issue a gauge transformation, that we recall below, is introduced to remove the bad term $2|u|^{2} u_{x}$.

For $f \in L^{2}(\mathbb{T})$, let

$$
G(f)(x):=\exp (-i J(f)) f(x)
$$

where

$$
J(f)(x):=\frac{1}{2 \pi} \int_{0}^{2 \pi} \int_{\theta}^{x}|f(y)|^{2}-\frac{1}{2 \pi}\|f\|_{L^{2}(\mathbb{T})}^{2} d y d \theta .
$$

Note $G(f)$ is $2 \pi$-periodic since its integrand has zero mean value. Then for $u \in C\left([-T, T] ; L^{2}(\mathbb{T})\right)$

$$
\mathcal{G}(u)(t, x):=G(u(t))(x-2 t m(u)) .
$$

Note the $L^{2}$ norm of $\mathcal{G}(u)(t, x)$ is still conserved since the torus is invariant under translation. We have that

$$
\mathcal{G}: C\left([-T, T] ; H^{\sigma}(\mathbb{T})\right) \rightarrow C\left([-T, T] ; H^{\sigma}(\mathbb{T})\right)
$$

is a homeomorphism for any $\sigma \geq 0$ and locally bi-Lipschitz on subsets of $C\left([-T, T] ; H^{\sigma}(\mathbb{T})\right)$ with prescribed $\|u(0)\|_{L^{2}}$.

Then if $u$ is a solution to $\operatorname{DNLS}(2.1)$ and $v:=\mathcal{G}(u)$, then $v$ solves the gauged DNLS equation GDNLS:

$$
v_{t}-i v_{x x}=-v^{2} \bar{v}_{x}+\frac{i}{2}|v|^{4} v-i \psi(v) v-i m(v)|v|^{2} v
$$

with initial data $v(0)=\mathcal{G}(u(0))$, where

$$
\begin{array}{rlr}
m(v)(t) & :=\frac{1}{2 \pi} \int_{\mathbb{T}}|v(x, t)|^{2} d x & \text { and } \\
\psi(v)(t) & :=-\frac{1}{\pi} \int_{\mathbb{T}} \operatorname{Im}\left(v \bar{v}_{x}\right) d x+\frac{1}{4 \pi} \int_{\mathbb{T}}|v|^{4} d x-m(v)^{2} .
\end{array}
$$

Note that $m(v)$ is conserved in time; more precisely $m(v)(t)=\frac{1}{2 \pi} \int_{\mathbb{T}}|v(x, 0)|^{2} d x=m(u)$, and that both $m(v)$ and $\psi(v)$ are real. Now we show how the energy $E(u)$ and $H(u)$ transform under the gauge. We have

$$
\begin{aligned}
H(u) & =\operatorname{Im} \int_{\mathbb{T}} u \bar{u}_{x} d x+\frac{1}{2} \int_{\mathbb{T}}|u|^{4} d x \\
& =\operatorname{Im} \int_{\mathbb{T}} v \bar{v}_{x}-\frac{1}{2} \int_{\mathbb{T}}|v|^{4} d x+2 \pi m^{2}=: \mathscr{H}(v)
\end{aligned}
$$

and

$$
E(u)=\int v_{x} \overline{v_{x}} d x-\frac{1}{2} \operatorname{Im} \int v^{2} \overline{v v_{x}} d x+2 m \operatorname{Im} \int v \overline{v_{x}} d x-\frac{1}{2} m \int|v|^{4} d x+2 \pi m^{3}
$$


If we define

$$
\mathscr{E}(v):=\int_{\mathbb{T}}\left|v_{x}\right|^{2} d x-\frac{1}{2} \operatorname{Im} \int_{\mathbb{T}} v^{2} \bar{v} \overline{v_{x}} d x+\frac{1}{4 \pi}\left(\int_{\mathbb{T}}|v(t)|^{2} d x\right)\left(\int_{\mathbb{T}}|v(t)|^{4} d x\right),
$$

then $E(u)$ can be rewritten as

$$
E(u)=\mathscr{E}(v)+2 m \mathscr{H}(v)-2 \pi m^{3}=: \mathcal{E}(v) .
$$

Unfortunately while gauging the DNLS equation one looses track of the key features of the Gibbs measure associated to the original equation since, the gauge transformation mixes the Fourier coefficients in a way that is hard to control. Our approach in [54] then can be summarized in the following steps.

Step 1: We consider directly the gauged problem (2.3) and we define a new Gibbs measure $d \mu$ for this problem using its energy truncated $(v)$ in (2.8). Informally

$$
\text { "d } \mu=\frac{1}{Z} e^{-\mathcal{E}(v)-m(v)} d v ",
$$

and its support is in spaces that scale like $H^{s}, s<\frac{1}{2}$. To define this measure as usual we truncate the initial value problem (2.3) on a frequency window of size $N$ and we work with the corresponding finite dimensional system. Local well-posedness, independent of $N$, of this truncated problem in the support of the measure is not an issue, and in fact randomness is not necessary ${ }^{2}$ to establish it. But there are two main difficulties that need to be resolved in order to define $d \mu$. The first is that the truncated initial value problem coming from (2.3) is no longer in Hamiltonian form and hence one has to prove directly that its flow preserves volumes. This is done in Lemma 4.1 in [54]. The second difficulty is that the energy $\mathcal{E}\left(v_{N}\right)$, where $v_{N}$ is the solution to the truncated problem, is no longer invariant. Hence $d \mu_{N}$, the truncated version of $d \mu$, is not invariant. This is contrary to what happens for example in [63], for the un-gauged truncated initial DNLS problem.

Step 2: In this step we show that $\mathcal{E}\left(v_{N}\right)$ is almost conserved and hence $d \mu_{N}$ is almost invariant. This is the content of Theorem 4.2 in [54].

Step 3: In this step we put all the ingredients together such as local well-posedness, almost invariance of the measure and the argument of Bourgain to move from local to global solutions. Finally we take the limit as $N$ tends to infinity and we also show that although $d \mu_{N}$ was only almost invariant, its limit $d \mu$ is invariant. This step is the content of the fundamental Lemma 6.1, Proposition 6.2 and Theorem 6.3 in [58].

Here we give a very schematic rendering of the main idea contained in Lemma 6.1 that allows us to move from local to global well-posedness. Let us call $\Phi_{N}$ the flow of the truncated initial value problem (2.3). Let us assume that the initial data are in a set $C_{N}(K)$ of (truncated) initial data, contained in the support of the measure, with norms bounded by $K$, where $K$ is large. If the initial data of the truncated problem are in $C_{N}(K)$ then one has local well-posed on a time interval of length $\delta \sim K^{-\gamma}$, where $\gamma>0$ is independent of $N$. Furthermore, from the definition of $d \mu_{N}$ from Step 1 one has the large deviation estimate

$$
\mu_{N}\left(C_{N}(K)\right) \leq e^{-K^{2}}
$$

\footnotetext{
${ }^{2}$ Here deterministic local well-posedness results of [33] in Fourier Lebesgue spaces $\mathcal{F} L^{s, r}$ suffice
} 
We then fix a large time $T$ and we define the subset of initial data $\widetilde{\Omega}_{N}$ by

$$
\widetilde{\Omega}_{N}:=\left\{v_{0}^{N}: \Phi_{N}(j \delta)\left(v_{0}^{N}\right) \in C_{N}(K), j=0,1, \cdots\left[\frac{T}{\delta}\right]\right\} .
$$

Note that $\widetilde{\Omega}_{N}^{c}=\bigcup_{k=0}^{\left[\frac{T}{\delta}\right]} D_{k}$, where

$$
\begin{aligned}
D_{k} & =\left\{v_{0}^{N} ; k=\min \left\{j: \Phi_{N}(j \delta)\left(v_{0}^{N}\right) \in C_{N}(K)^{c}\right\}\right\}, \\
& \left.=\left[\bigcap_{j=0}^{k-1} \Phi_{N}(-j \delta) C_{N}(K)\right)\right] \cap \Phi_{N}(-k \delta)\left(C_{N}(K)^{c}\right) .
\end{aligned}
$$

One verifies easily that the sets $D_{k}$ satisfy

$$
D_{0}=C_{N}(K)^{c}, \quad D_{k}=C_{N}(K) \cap \Phi_{N}(-\delta)\left(D_{k-1}\right) .
$$

From the almost conservation of the energy mentioned in Step 2, iterating in $k \in\left\{0, \ldots\left[\frac{T}{\delta}\right]\right\}$, one obtains

$$
\mu_{N}\left(D_{K}\right) \leq e^{c(\delta) N^{-\beta} K^{8}} \mu_{N}\left(D_{K-1}\right) \leq e^{k c(\delta) N^{-\beta} K^{8}} e^{-c K^{2}}
$$

and thus

$$
\mu_{N}\left(\widetilde{\Omega}_{N}^{c}\right) \leq \sum_{k=0}^{\left[\frac{T}{\delta}\right]} e^{k c(\delta) N^{-\beta} K^{8}} e^{-c K^{2}} \lesssim\left[\frac{T}{\delta}\right] e^{-c K^{2}} \sim T K^{\gamma} e^{-c K^{2}},
$$

for $N \geq N_{0}(T, K)$. By choosing $K \sim\left(\log \frac{T}{\varepsilon}\right)^{\frac{1}{2}}$, one has $\mu_{N}\left(\widetilde{\Omega}_{N}^{c}\right)<\varepsilon$ which implies that for a fixed $T$ one can find a set of initial data $\widetilde{\Omega}_{N}$ of almost full measure which evolution spans the whole interval of time $[0, T]$.

One my wonder though what is the connection between the measure $d \nu$ in [63] for the DNLS and the measure $d \mu$ above for the GDNLS. We answered this question in [57], by showing that indeed the two measures are absolutely continuous with respect to each other. We discuss this in the next section because the topic is relevant on its own terms.

\section{Gaussian measures under gauge transformations}

In [57] we prove the absolute continuity of Brownian bridges under certain type of gauge transformations. More precisely we give a proof of the absolute continuity between Gaussian measures naturally associated with complex Brownian motions and their images under certain gauge transformations, which could be of anticipative type and hence not under the scope of Girsanov's theorem [60]. The general nature of our theorem as well as the ubiquitous role of gauge transformations in the study of dispersive and wave equations ${ }^{3}$ should make the type of result in [57] applicable in other situations as well. The motivation arises from the work in [54] described above on the periodic DNLS. In that work a weighted Wiener measure of the form $\mu=Z^{-1} \exp \left(-\int_{\mathbb{T}} \mathcal{N}(v)\right) \cdot \rho$, where $\rho$ is the Gaussian measure, is constructed and its invariance established for the associated gauged equation. Here $\mathcal{N}(v)$ denotes the nonlinear terms in the energy $\mathcal{E}$ of GDNLS. The question arises as to whether the ungauged measure $\tilde{\mu}=\mu \circ \mathcal{G}$, which is also invariant as we will see below, is also absolutely continuous with respect

\footnotetext{
${ }^{3}$ For many equations gauge transformations are essential to convert the nonlinearity into a better one where resonant interactions are manageable.
} 
to $\nu$, the weighted Wiener measure that was constructed by Thomann-Tzvetkov in [63] directly from the (ungauged) periodic DNLS. In [57] we answered this question in the affirmative and thus in particular showed the measure constructed in [63] is indeed invariant under the flow of periodic DNLS.

There is a vast literature on Gaussian measures under nonlinear transformations both of non-anticipative and anticipative type (see eg. [7, 10, 16, 17, 29, 36, 51, 62] and other references therein). Their general framework however does not fit those gauge transformations we have in mind. The work of Cambronero-McKean [16] on the periodic KdV and the Miura gauge transformation- exploited by Quastel and Valkó [61]- is not directly applicable either. The absolute continuity of Gaussian measures in the context of periodic PDE is an intricate problem that requires tools from stochastic analysis and probability. It is intimately connected with how Gaussian measures and their supports transform under gauge transformations as well as other nonlinear transformation, in the periodic setting. This is a problem with interest in its own right and the subject of current research.

To recap then, from the previous Section 2, the output from [54] gives the following:

Theorem 3.1 (Almost sure global well-posedness of GDNLS) There exists a set $\Omega, \mu\left(\Omega^{c}\right)=0$ such that for every $v_{0} \in \Omega$ the IVP GDNLS with initial data $v_{0}$ is globally well-posed.

Theorem 3.2 (Invariance of $\mu$ ) The measure $\mu$ is invariant under the flow $\Phi(t)$ of GDNLS

The last step is going back to the ungauged DNLS equation. By pulling back the gauge, it follows easily from Theorems 3.1 and 3.2 that we have invariance of the ungauged measure $\tilde{\mu}:=\mu \circ \mathcal{G}$.

Recall, $\mu$ is a measure on $\Omega$ and $\mathcal{G}^{-1}: \Omega \rightarrow \Omega$ measurable. The measure $\tilde{\mu}$ is defined then by

$$
\tilde{\mu}(A):=\mu(\mathcal{G}(A))=\mu\left(\left\{v: \mathcal{G}^{-1}(v) \in A\right\}\right) .
$$

for all measurable sets $A$ or equivalently - for integrable $F$ - by

$$
\int F d \tilde{\mu}=\int F \circ \varphi d \mu
$$

Theorem 3.3 (Almost sure global well-posedness of DNLS) There exists a subset $\Sigma$ of the Fourier-Lebesgue space $\mathcal{F}^{\frac{2}{3}-, 3}(\mathbb{T})$ with $\tilde{\mu}\left(\Sigma^{c}\right)=0$ such that for every $u_{0} \in \Sigma$ the IVP DNLS with initial data $u_{0}$ is globally well-posed.

Finally we show that the measure $\tilde{\mu}$ is invariant under the flow map of DNLS.

Theorem 3.4 (Invariance of measure under DNLS flow) The measure $\tilde{\mu}=\mu \circ \mathcal{G}$ is invariant under the DNLS flow.

We are now ready to ask: what is $\tilde{\mu}=\mu \circ \mathcal{G}$ really? Is it absolutely continuous with respect to $\nu$, the measure that can be naturally constructed for DNLS by using its energy $E$,

$$
E(u)=\int_{\mathbb{T}}\left|u_{x}\right|^{2} d x+\frac{3}{2} \operatorname{Im} \int_{\mathbb{T}} u^{2} \overline{u u_{x}} d x+\frac{1}{2} \int_{\mathbb{T}}|u|^{6} d x
$$

\footnotetext{
${ }^{4}$ For a definition of Fourier Lebesgue spaces see for example [54]. In particular, $\mathcal{F} L^{\frac{2}{3}-, 3}(\mathbb{T})$ scales like $H^{\frac{1}{2}-}(\mathbb{T})$.
} 


$$
=: \quad \int_{\mathbb{T}}\left|u_{x}\right|^{2} d x+\mathcal{K}(u)
$$

as done by Thomann-Tzevtkov in [63]?

We know $\tilde{\mu}$ is invariant and that the ungauged DNLS equation is globally well-posed almost surely with respect to $\tilde{\mu}$. Treating the weight is easy. The problem is un-gauging the Gaussian measure $\rho$.

Hence the question becomes: what is $\tilde{\rho}:=\rho \circ \mathcal{G}$ ? Is its restriction to a sufficiently small ball in $L^{2}$ absolutely continuous with respect to $\rho$ ? If so, what is its Radon-Nikodym derivative? We would like to compute $\tilde{\rho}$ explicitly. This turns out to be an intricate problem that requires tools from stochastic analysis and probability.

\subsection{Absolute continuity of Brownian bridges under gauge transformations}

There is an analytic theory on Gaussian measures under nonlinear transformations both of non-anticipative and anticipative type (see eg. [7] and references therein).

This theory is fairly well understood for transformations of the form $x+F(x)$ with $F$ a transformation from a Banach space (associated to the support of the measure) into a Hilbert space $H$, known as the Cameron-Martin space (associate to the construction of measure; $H=$ $\dot{H}^{1}$ in the case of DNLS above) and whose (Fréchet) derivative $F^{\prime}$ in the direction of $H$ exists and is 'nice', for example $\left.F^{\prime}\right|_{H}$ is Hilbert-Schmidt. But this framework does not fit (directly) the gauge transformations as the one above. Gauge transformations as the ones used above are $L^{2}$ unitary transformations which do not have this $I+F$ form. The work of Cambronero-McKean on the periodic KdV and the Miura gauge transformation- exploited by Quastel and Valkó- is not directly applicable either.

To further understand the ungauged measure one should stop thinking about the solution $v$ as a infinite dimension vector of Fourier modes and start thinking instead about $v$ as a (periodic) complex Brownian path in $\mathbb{T}$ (Brownian bridge) solving a certain stochastic process.

We recall that to ungauge we need to define

$$
\mathcal{G}^{-1}(v)(x):=\exp (i J(v)) v(x)
$$

where $J(v)$ is defined as in (2.2).

It will be important later that $J(v)(x)=J(|v|)(x)$. Then, if $v$ satisfies

$$
d v(x)=\underbrace{d B(x)}_{\text {Brownian motion }}+\underbrace{b(x) d x}_{\text {drift terms }}
$$

by Ito's calculus, and since $\exp (i J(v))$ is differentiable, we have:

$$
d \mathcal{G}^{-1} v(x)=\exp (i J(v)) d v+i v \exp (i J(v))\left(|v(x)|^{2}-\frac{1}{2 \pi}\|v\|_{L^{2}}^{2}\right) d x+\ldots
$$

Substituting above one has

$$
\left.d \mathcal{G}^{-1} v(x)=\exp (i J(v))[d B(x)+a(v, x, \omega)) d x\right]+\ldots
$$

where

$$
a(v, x, \omega)=i v\left(|v(x)|^{2}-\frac{1}{2 \pi}\|v\|_{L^{2}}^{2}\right) .
$$


The fact that $\exp (i J(v))$ is a unitary operator is crucial. If one could now prove Novikov's condition:

$$
\mathbb{E}\left[\exp \left(\frac{1}{2} \int a^{2}(v, x, \omega) d x\right)\right]<\infty
$$

then one could apply Girsanov's theorem ${ }^{5}$ to finish off. However, Girsanov's theorem doesn't save the day quite yet. If one reads the theorem carefully one realizes that an important condition is that $a(v, x, \omega)$ is non-anticipative; in the sense that it only depends on the Brownian motion $v(x)$ up to 'time' $x$ and not further. This however is not true in our case. The drift term $a(v, x, \omega)$ in (3.1) involves the $L^{2}$ norm of $v(x)$ and hence it is anticipative. A different strategy is needed. We use in fact the conformal invariance of complex Brownian motions, together with 'linearization'. We now give some details.

If $W(t)=W_{1}(t)+i W_{2}(t)$ is a complex Brownian motion, and $\phi$ is an analytic function, then $Z=\phi(W)$ is, after a suitable time change, again a complex Brownian motion. In what follows, let $Z(t)$ play the role of our complex Brownian motion $v(x)$, and let $\phi$ be the exponential function. For $Z(t)=\exp (W(s))$ the time change is given by

$$
t=t(s)=\int_{0}^{s}\left|e^{W(r)}\right|^{2} d r, \quad \frac{d t}{d s}=\left|e^{W(s)}\right|^{2},
$$

or equivalently

$$
s(t)=\int_{0}^{t} \frac{d r}{|Z(r)|^{2}}, \quad \frac{d s}{d t}=\frac{1}{|Z(t)|^{2}} .
$$

We are interested in $Z(t)$ in the interval $0 \leq t \leq 2 \pi$, and thus we introduce the stopping time

$$
\mathcal{S}=\inf \left\{s ; \int_{0}^{s}\left|e^{W(r)}\right|^{2} d r=2 \pi\right\} .
$$

It is important to note that the stopping time $\mathcal{S}$ depends only on the real part $W_{1}(s)$ of $W(s)$, (or equivalently only on $|Z|$ ).

If we write $Z(t)$ in polar coordinate $Z(t)=|Z(t)| e^{i \Theta(t)}$ we have

$$
W(s)=W_{1}(s)+i W_{2}(s)=\log \mid Z(t(s) \mid+i \Theta(t(s))
$$

and $W_{1}$ and $W_{2}$ are real independent Brownian motions. If we define

$$
\begin{aligned}
\tilde{W}(s) & :=W_{1}(s)+i\left[W_{2}(s)+\int_{0}^{t(s)} h(|Z|)(r) d r\right] \\
& =W_{1}(s)+i\left[W_{2}(s)+\int_{0}^{t(s)} h\left(e^{W_{1}}\right)(r) d r,\right]
\end{aligned}
$$

where essentially $\quad h(|Z|)(\cdot)=|Z(\cdot)|^{2}-\|Z\|_{L^{2}}^{2}$, we then have

$$
e^{\tilde{W}(s)}=\mathcal{G}^{-1}(Z)(t(s)) \text {. }
$$

In terms of $W$, the gauge transformation is now easy to understand. It gives a complex process such that:

\footnotetext{
${ }^{5}$ Roughly, Girsanov's theorem states that if we change the drift coefficient of a given Ito process in an appropriate way, then the law of the process will not change dramatically. In fact the new process law will be absolutely continuous with respect to the law of the original process and we can compute explicitly the Radon-Nikodym derivative.
} 
- The real part is left unchanged.

- The imaginary part is translated by the function $J(Z)(t(s))$ which depends only on the real part (ie. on $|Z|$, which has been fixed) and in that sense is deterministic.

- It is now possible to use Cameron-Martin-Girsanov's theorem only for the law of the imaginary part and conclude:

We can now thus conclude. If $\eta$ denotes the probability distribution of $W$ and $\tilde{\eta}$ the distribution of $\tilde{W}$ we have the absolute continuity of $\tilde{\eta}$ and $\eta$, whence the absolute continuity between $\tilde{\rho}$ and $\rho$ follows, with the same Radon-Nikodym derivative (re-expressed back in terms of $t$ ).

All in all then we prove that our ungauged measure $\nu$ is in fact essentially (up to normalizing constants) of the form

$$
d \tilde{\mu}(u)=\chi_{\|u\|_{L^{2}} \leq B} e^{-\mathcal{K}(u)} d \rho=d \nu(u),
$$

the weighted Wiener measure associated to DNLS (constructed by Thomann-Tzvetkov). In particular we prove its invariance.

We need to remark at this point that the above argument needs to be done carefully for complex Brownian bridges (periodic Brownian motions) by conditioning properly. Note that $W(s)$ is a Brownian motion conditioned to end up at the same place when the total variation time $t=t(s)$ reaches $2 \pi$. The time when this occurs is our $\mathcal{S}$. For more details see [57].

\section{The periodic quintic NLS equation in $3 \mathrm{D}$ and the periodic Navier-Stokes equa- tion in $2 \mathrm{D}$ and $3 \mathrm{D}$}

In this section we show how, inspired by the original work of Bourgain in $[8,9]$, one can show that randomization of the initial data can give the existence of a local flow for data that are mass or energy supercritical, or in a sense equivalently, the existence of a set of global solutions with data of infinite mass or energy.

4.1 The periodic quintic NLS equation in 3D

In this subsection we summarize the work in [58]. The quintic NLS in 3D is an energy critical equation, that is the $H^{1}$ norm of the solution is invariant with respect to the parabolic scaling of this equation. In both $\mathbb{R}^{3}[18]$ and $\mathbb{T}^{3}$ [35] global well-posedness for initial data in $H^{s}, s \geq 1$ has been proved. On the other hand if the data have infinite energy the problem becomes supercritical and the question of global well-posedness is still open. In the work we are summarizing below we demonstrate that for both the focusing and defocusing case, we can equip the energy supercritical set of data $H^{1-\epsilon}\left(\mathbb{T}^{3}\right)$ with a certain probability measure such for data in a subset of almost full measure a local flow is well defined. This is Theorem (4.1) below ${ }^{6}$. This kind of statement is similar to that proved in the first part of [9]. In the second part of that paper,

\footnotetext{
${ }^{6}$ On the other hand one can rephrase this theorem by saying that for a fixed large time $T$ there is a subset $\Sigma$ of nonzero measure for which any data in $\Sigma$ evolves into a solution that lives for the whole interval $[0, T]$. This shows the existence of large data global solutions with infinite energy.
} 
Bourgain was able to extend the result from local to global, ultimately showing that there is a set of initial data of full measure evolving for an arbitrarily long interval of time. He proved this using the invariance of the probability measure, which in his case was the Gibbs measure. Unfortunately in our case the Gibbs measure lives in $\mathrm{H}^{-1 / 2-}$, a space that is way too rough to conduct any type of well-posedness analysis, hence we cannot extend a local in time result to a global one, at least not using the invariance of the measure ${ }^{7}$.

We consider then the Cauchy initial value problem,

$$
\left\{\begin{array}{l}
i u_{t}+\Delta u=\lambda u|u|^{4} \quad x \in \mathbb{T}^{3} \\
u(0, x)=\phi(x)
\end{array}\right.
$$

where $\lambda= \pm 1$. We also stress the fact that here $\mathbb{T}^{3}$ is a rational torus, in the sense that if $\left(T_{1}, T_{2}, T_{3}\right)$ are the periods along the three directions, then there exists $\left(n_{1}, n_{2}, n_{3}\right) \in \mathbb{Z}^{3}$ such that $\left(T_{1}, T_{2}, T_{3}\right) \cdot\left(n_{1}, n_{2}, n_{3}\right)=0$. This is an important assumption since it implies that the solution $S(t) \phi(x)$ to the linear problem associated to (4.1) is also periodic in time, a fact that is used heavily. In fact without loss of generality we will assume that $\left(T_{1}, T_{2}, T_{3}\right)=(1,1,1)$.

Our setting to show almost sure local well posedness is similar to that of Bourgain in [9]. More precisely, we consider data $\phi \in H^{1-\varepsilon}\left(\mathbb{T}^{3}\right)$ for any $\varepsilon>0$ of the form

$$
\phi(x)=\sum_{n \in \mathbb{Z}^{3}} \frac{1}{(1+|n|)^{\frac{5}{2}}} e^{i n \cdot x}
$$

whose randomization according to (1.2) is

$$
\phi^{\omega}(x)=\sum_{n \in \mathbb{Z}^{3}} \frac{g_{n}(\omega)}{(1+|n|)^{\frac{5}{2}}} e^{i n \cdot x} .
$$

The main result in [58] is on almost sure local well-posedness and can then be stated as follows,

Theorem 4.1 Let $\phi$ as in (4.2). Then there exist $s>1,0<\delta_{0} \ll 1$ and $r=r(s, \alpha)>0$ such that for any $\delta<\delta_{0}$, there exists $\Omega_{\delta} \in A$ with

$$
\mathbb{P}\left(\Omega_{\delta}^{c}\right)<e^{-\frac{1}{\delta^{r}}},
$$

and for each $\omega \in \Omega_{\delta}$ there exists a unique solution $u$ of (4.1) in the space

$$
S(t) \phi^{\omega}+X^{s}([0, \delta))_{d}
$$

where $S(t) \phi^{\omega}$ is the linear evolution of the initial data $\phi^{\omega}$ given by (4.3).

One can also reformulate this theorem to prove long time existence of large data with infinite energy. Namely one can prove the following theorem.

Theorem 4.2 Assume $s$ as above. For fixed $T$ there exists a set $\Omega_{T} \in A$ with

$$
\mathbb{P}\left(\Omega_{T}\right) \geq \epsilon_{T}>0,
$$

and for each $\omega \in \Omega_{T}$ there exists a unique solution $u$ of (4.1) in the space

$$
S(t) \phi^{\omega}+X^{s}([0, T))_{d},
$$

\footnotetext{
${ }^{7}$ In fact almost sure global well-posedness for energy supercritical solutions for the periodic quintic NLS equation in $3 \mathrm{D}$ is still open.
} 
where $S(t) \phi^{\omega}$ is the linear evolution of the initial data $\phi^{\omega}$ given by (4.3).

Here the space $X^{s}([0, \delta))_{d}$ is a metric space of Sobolev regularity $s$ which definition can be found in [58], the metric $d$ comes from (4.8) below. We note that this space is not based on the usual $X^{s, b}$ defined below, but the proof of Theorem 4.1 and Theorem 4.2 can be performed, even in a easier way, in $X^{s, 1 / 2}$. In fact below we will show some estimates in this space in order to give an idea of the type of arguments that are needed to prove the theorems above.

Definition 4.3 We define the space $X^{s, b}\left(\mathbb{T} \times \mathbb{T}^{3}\right)$ via its norm:

$$
\|f\|_{X^{s, b}}:=\left(\sum_{\lambda} \sum_{n \in \mathbb{Z}^{3}}|\hat{f}(\lambda, n)|^{2}\left(1+|n|^{2}\right)^{s}\left(1+\left.|\lambda-| n\right|^{2} \mid\right)^{2 b}\right)^{1 / 2} .
$$

To prove Theorem 4.1 we proceed in two steps. First we consider the initial value problem

$$
\left\{\begin{array}{l}
i v_{t}+\Delta v=\mathcal{N}(v) \quad x \in \mathbb{T}^{3} \\
v(0, x)=\phi^{\omega}(x)
\end{array}\right.
$$

where

$$
\mathcal{N}(v):=\lambda\left(v|v|^{4}-3 v\left(\int_{\mathbb{T}^{3}}|v|^{4} d x\right)\right)
$$

with $\lambda= \pm 1$ and $\phi^{\omega}(x)$ the initial datum as in (4.3). Introducing the nonlinearity $\mathcal{N}(v)$ is similar to the Wick ordering used in [9] and it is needed in order to exploit the independence of the random variables as we will see below. To make the notation simpler set

$$
\beta_{v}(t)=3 \int_{\mathbb{T}^{3}}|v|^{4} d x
$$

and define

$$
u(t, x):=e^{i \lambda \int_{0}^{t} \beta_{v}(s) d s} v(t, x) .
$$

We observe that $u$ solves the initial value problem (4.1) with datum $\phi^{\omega}$. Now suppose that one obtains well-posedness for the initial value problem (4.4) in a certain Banach space $(X,\|\cdot\|)$ then one can transfer those results to the initial value problem (4.1) by using a metric space $X_{d}:=(X, d)$ where

$$
d(u, v):=\left\|e^{-i \lambda \int_{0}^{t} \beta_{u}(s) d s} u(t, x)-e^{-i \lambda \int_{0}^{t} \beta_{v}(s) d s} v(t, x)\right\| .
$$

We now summarize the steps needed in order to show local well-posedness for the initial value problem (4.4).

Step1: We define $w:=v-S(t) \phi^{\omega}(x)$ and we consider the initial value problem ${ }^{8}$ satisfied by $w$, that is

$$
\left\{\begin{array}{l}
i w_{t}+\Delta w=\mathcal{N}\left(w+S(t) \phi^{\omega}(x)\right) \quad x \in \mathbb{T}^{3} \\
w(0, x)=0
\end{array}\right.
$$

The goal is to show that (4.9) can be studied in $X^{s, 1 / 2}$ for some $s>1$ in spite of the fact that $S(t) \phi^{\omega}(x)$ is in $H^{1-\epsilon}$ for each fixed time.

\footnotetext{
${ }^{8}$ Since we are only interested on the local well-posedness we can just assume that $\lambda=1$.
} 
Step 2: We set up the contraction method by defining the map

$$
\Phi(w)=\chi_{[-\delta, \delta]}(t) \int_{0}^{t} S\left(t-t^{\prime}\right) \mathcal{N}\left(w+S\left(t^{\prime}\right) \phi^{\omega}(x)\right) d t^{\prime},
$$

where $\chi_{[-\delta, \delta]}(t)$ is a smooth characteristic function of $[-\delta, \delta]$ for $\delta$ small. The goal is to show that

$$
\|\Phi(w)\|_{X^{s, 1 / 2}} \lesssim C+\delta^{\alpha}\|w\|_{X^{s, 1 / 2}}^{5}
$$

and

$$
\left\|\Phi\left(w_{1}\right)-\Phi\left(w_{2}\right)\right\|_{X^{s, 1 / 2}} \lesssim \delta^{\alpha}\left(\left\|w_{1}-w_{2}\right\|_{X^{s, 1 / 2}}\right)
$$

We now show two extreme estimates that one has to perform in order to prove (4.10) and (4.11). The first estimate concern the case when all terms in $\mathcal{N}\left(w+S(t) \phi^{\omega}(x)\right)$ are deterministic, that is we consider $\mathcal{N}(w)$. In this case we can use the arguments in [34] to conclude. In this argument one uses duality and estimates an integral of six functions by splitting it into two trilinear $L^{2}$ integrals. One obtains

$$
\left\|\chi_{[-\delta, \delta]}(t) \int_{0}^{t} S\left(t-t^{\prime}\right) \mathcal{N}\left(w\left(t^{\prime}\right)\right) d t^{\prime}\right\|_{X^{s, 1 / 2}} \lesssim \delta^{\alpha}\|w\|_{X^{s, 1 / 2}}^{5} .
$$

Note that in [34] the authors consider the full quintic nonlinearity, not just $\mathcal{N}(w(t))$, and they are in a more difficult set up, that is in their case $s=1$, the energy critical case. Because of this critical situation they have to use more subtle spaces than the $X^{s, 1 / 2}$, but the basic arguments are the same.

Next we consider the case when all terms in $\mathcal{N}\left(w+S(t) \phi^{\omega}(x)\right)$ are random, that is we consider $\mathcal{N}\left(S(t) \phi^{\omega}(x)\right)$. Let us not worry about having to restrict the time interval to $[-\delta, \delta]$, this can be taken care of with standard argument later. Let us recall that [45]

$$
\left\|\int_{0}^{t} S\left(t-t^{\prime}\right) F\left(t^{\prime}\right) d t^{\prime}\right\|_{X^{s, 1 / 2}} \lesssim\|F\|_{X^{s,-1 / 2}} .
$$

Then by duality we need to estimate

$$
\int_{\mathbb{T}} \int_{\mathbb{T}^{3}} D^{s} \mathcal{N}\left(S(t) \phi^{\omega}(x)\right) k(t, x) d t d x
$$

where $D^{s}$ is the symbol for the $s$ derivative and $k(t, x) \in X^{0,1 / 2}$. Let us also assume that $k(t, x)$ is localized in frequency in a dyadic block of size $N$ and all the other functions are localized in dyadic blocks of size $N_{i}$ respectively, and $N_{1} \sim N>>N_{2} \geq N_{3} \geq N_{4} \geq N_{5}$. Also in this case we pass from the $L^{1}$ norm in (4.12) to two trilinear expressions in $L^{2}$ where we match $N, N_{4}, N_{5}$ and $N_{1}, N_{2}, N_{3}$. We also assume that $\left.|\lambda-| n\right|^{2} \mid \lesssim N^{\alpha}$ for $\alpha<1 / 2$ since otherwise one can use Strichartz estimates and the fact that $S(t) \phi^{\omega}(x)$ are in better $L^{p}$ spaces to conclude. Here we only estimate the square of the second trilinear expression with the operator $D^{s}$ acting on $N_{1}$, which can be written as

$$
\sum_{\lambda} \sum_{n \in \mathbb{Z}^{2}} N^{2 s}\left|\sum_{S_{\lambda, n}} \frac{g_{n_{1}}(\omega)}{\left(1+\left|n_{1}\right|\right)^{5 / 2}} \frac{\bar{g}_{n_{2}}(\omega)}{\left(1+\left|n_{2}\right|\right)^{5 / 2}} \frac{g_{n_{3}}(\omega)}{\left(1+\left|n_{3}\right|\right)^{5 / 2}}\right|^{2}
$$

where

$$
S_{\lambda, n}:=\left\{\begin{array}{cc}
n=n_{1}-n_{2}+n_{3} ; & n_{1}, n_{3}, \neq n_{2} \\
\lambda=\left|n_{1}\right|^{2}-\left|n_{2}\right|^{2}+\left|n_{3}\right|^{2}
\end{array}\right\}
$$


For the second trilinear estimate we can simply use again the argument in [34]. We now change variables by setting $\lambda-|n|^{2}=\eta$ and we integrate in $\eta$, where we recall $|\eta|<N^{\alpha}$. We have

$$
\sup _{\eta} \sum_{n \in \mathbb{Z}^{2}} N^{2 s+\alpha}\left|\sum_{S_{\eta, n}} \frac{g_{n_{1}}(\omega)}{\left(1+\left|n_{1}\right|\right)^{5 / 2}} \frac{\bar{g}_{n_{2}}(\omega)}{\left(1+\left|n_{2}\right|\right)^{5 / 2}} \frac{g_{n_{3}}(\omega)}{\left(1+\left|n_{3}\right|\right)^{5 / 2}}\right|^{2}
$$

where

$$
S_{\eta, n}:=\left\{\begin{array}{cc}
n=n_{1}-n_{2}+n_{3} ; & n_{1}, n_{3}, \neq n_{2} \\
\eta=\left|n_{1}\right|^{2}-\left|n_{2}\right|^{2}+\left|n_{3}\right|^{2}-|n|^{2}
\end{array}\right\} .
$$

Now we use a large deviation estimate and the independence of the random variables to claim that for $\omega \in \tilde{\Omega}$, with $\mathbb{P}\left(\tilde{\Omega}^{c}\right) \leq e^{-1 / \delta}$ we can continue with

$$
\begin{aligned}
& \lesssim \delta^{-m} \sup _{\eta} \sum_{n \in \mathbb{Z}^{2}} N^{2 s+\alpha} \int_{\Omega}\left|\sum_{S_{\eta, n}} \frac{g_{n_{1}}(\omega)}{\left(1+\left|n_{1}\right|\right)^{5 / 2}} \frac{\bar{g}_{n_{2}}(\omega)}{\left(1+\left|n_{2}\right|\right)^{5 / 2}} \frac{g_{n_{3}}(\omega)}{\left(1+\left|n_{3}\right|\right)^{5 / 2}}\right|^{2} d \omega \\
& \lesssim \delta^{-m} N^{2 s+\alpha} N_{1}^{-5} N_{2}^{-5} N_{3}^{-5}\left|S_{\eta}\right|
\end{aligned}
$$

where

$$
S_{\eta}=\left\{\begin{array}{c}
\left(n, n_{1}, n_{2}, n_{3}\right) / n=n_{1}-n_{2}+n_{3} ; \quad n_{1}, n_{3} \neq n_{2}, \\
\eta=\left|n_{1}\right|^{2}-\left|n_{2}\right|^{2}+\left|n_{3}\right|^{2}-|n|^{2}
\end{array}\right\}
$$

Now we fix $n_{2}, n_{3}$, we replace $n=n_{1}-n_{2}+n_{3}$ in the quadratic equation and we note that $n_{1}$ belongs to a plane, hence there are $N_{1}^{2}$ many frequencies. We then continue the estimate with

$$
\lesssim \delta^{-m} N^{2 s+\alpha} N_{1}^{2-5} N_{2}^{3-5} N_{3}^{3-5} \lesssim \delta^{-m} N^{2 s-3+\alpha}
$$

and hence we want $s<\frac{3-\alpha}{2}$.

Remark 4.4 We note that the condition $n_{1}, n_{3} \neq n_{2}$ is used both for the independence of the random variable and to claim that for fixed $n_{2}, n_{3}$ the frequency $n_{1}$ satisfies a linear relationship, namely $<n_{1}, n_{2}-n_{3}>=C$.

To obtain the full Theorem 4.1 one needs to consider many combinations of random and nonrandom trilinear estimates but we will not do this here.

4.2 The periodic 2D and 3D Navier-Stokes equations: randomization of the initial data and the Galerkin approximation scheme

In this section we explore the consequences of randomizing initial data when we consider the Navier-Stokes equation. We recall that for this equation both in 2D and 3D existence of global weak solutions is proved in $L^{2}$, where the energy is conserved. In $2 \mathrm{D}$ uniqueness is also proved. Here we show that by randomizing the initial data in $H^{-\alpha}$, for certain $\alpha>0$, one can still claim existence of global weak solutions, and in 2D also uniqueness. Note that in both 2D and 3D the space $H^{-\alpha}$ is supercritical. Due to the regularization properties of the heat flow and the fact that we are not using a fixed point theorem, but instead a Galerkin scheme, the estimates here are softer than the ones in the previous sections, see also [58] and [9]. The estimates used are in fact more similar to those in [14] for a certain wave equation. Below we recall the set up, the main results and we give an idea of the proofs. 
Consider the initial value problem for the incompressible Navier-Stokes equations given by

$$
\left\{\begin{array}{l}
\partial_{t} \vec{u}=\Delta \vec{u}-\mathbb{P} \nabla \cdot(\vec{u} \otimes \vec{u}) ; \quad x \in \mathbb{T}^{d}, t>0 \\
\nabla \cdot \vec{u}=0 \\
\vec{u}(x, 0)=\vec{f}(x)
\end{array}\right.
$$

where $f$ is divergence free and $\mathbb{P}$ is the Leray projection into divergence free vector fields given via

$$
\mathbb{P} \vec{h}=\vec{h}-\nabla \frac{1}{\Delta}(\nabla \cdot \vec{h}) .
$$

We now introduce the diagonal randomization of elements of $\left(H^{s}\left(\mathbb{T}^{d}\right)\right)^{d}$, which we will apply to our initial data. This is a multidimensional version of Definition 1.1.

Definition 4.5 [Diagonal randomization of elements in $\left.\left(H^{s}\left(\mathbb{T}^{d}\right)\right)^{d}\right]$ Let $\left(l_{n}(\omega)\right)_{n \in \mathbb{Z}^{d}}$ be a sequence of real, independent, random variables on a probability space $(\Omega, A, P)$. For $\vec{f} \in$ $\left(H^{s}\left(\mathbb{T}^{d}\right)\right)^{d}$, let $\left(\vec{a}_{n}^{i}\right), i=1,2, \ldots, d$, be its Fourier coefficients. We introduce the map from $(\Omega, A)$ to $\left(H^{s}\left(\mathbb{T}^{d}\right)\right)^{d}$ equipped with the Borel sigma algebra, defined by

$$
\omega \longrightarrow \vec{f}^{\omega}, \quad \vec{f}^{\omega}(x)=\left(\sum_{n \in \mathbb{Z}^{d}} l_{n}(\omega) \vec{a}_{n}^{1} e_{n}(x), \ldots, \sum_{n \in \mathbb{Z}^{d}} l_{n}(\omega) \vec{a}_{n}^{d} e_{n}(x)\right),
$$

where $e_{n}(x)=e^{i n \cdot x}$ and call such a map randomization.

The map (4.15) is measurable and $\vec{f}^{\omega} \in L^{2}\left(\Omega ;\left(H^{s}\left(\mathbb{T}^{d}\right)\right)^{d}\right)$, is an $\left(H^{s}\left(\mathbb{T}^{d}\right)\right)^{d}$-valued random variable. Also we remark again that such a randomization does not introduce any $H^{s}$ regularization (see Lemma B.1 in [14] for a proof of this fact), indeed $\left\|\overrightarrow{f^{\omega}}\right\|_{H^{s}} \sim\|\vec{f}\|_{H^{s}}$, but it gives improved $L^{p}$ estimates almost surely (see Proposition 4.10 below).

Remark 4.6 Since the Leray projection (4.14) can be written via its coordinates

$$
(\mathbb{P} \vec{h})_{j}=h_{j}+\sum_{k=1, \ldots, d} R_{j} R_{k} h_{k}
$$

where $\widehat{R_{j}(\phi)}(n)=\frac{i n_{j}}{|n|} \hat{\phi}(n), \quad n \in \mathbb{Z}^{d}$, one can easily see that the diagonal randomization defined in (4.15) commutes with the Leray projection $\mathbb{P}$.

Having defined a diagonal randomization, we need few more definitions in order to state the main results of this paper.

- Let $\mathcal{B}$ be a Banach space of functions. The space $C_{\text {weak }}((0, T), \mathcal{B})$ denotes the subspace of $L^{\infty}((0, T), \mathcal{B})$ consisting of functions which are weakly continuous, i.e. $v \in$ $C_{\text {weak }}((0, T), \mathcal{B})$ if and only if $\phi(v(t))$ is a continuous function of $t$ for any $\phi \in \mathcal{B}^{*}$.

- If $\left(X\left(\mathbb{T}^{d}\right)\right)^{d}$ denotes a space of vector fields on $\mathbb{T}^{d}$, we simply denote its norm by $\|\cdot\|_{X}$.

- We introduce an analogous notation to that of Constantin and Foias in [20]. In particular we write

$$
\begin{aligned}
H & =\text { the closure of }\left\{\vec{f} \in\left(C^{\infty}\left(\mathbb{T}^{d}\right)\right)^{d} \mid \nabla \cdot \vec{f}=0\right\} \text { in }\left(L^{2}\left(\mathbb{T}^{d}\right)\right)^{d}, \\
V & =\text { the closure of }\left\{\vec{f} \in\left(C^{\infty}\left(\mathbb{T}^{d}\right)\right)^{d} \mid \nabla \cdot \vec{f}=0\right\} \text { in }\left(H^{1}\left(\mathbb{T}^{d}\right)\right)^{d}, \\
V^{\prime} & =\text { the dual of } V .
\end{aligned}
$$


- We introduce some notation for the inner products in some of the spaces introduced above.

Given two vectors $\vec{u}$ and $\vec{v}$ in $\mathbb{R}^{d}$ we use the notation

$$
\langle\vec{u}, \vec{v}\rangle=\vec{u} \cdot \vec{v} .
$$

In $\left(L^{2}\left(\mathbb{T}^{d}\right)\right)^{d}$ we use the inner product notation

$$
(\vec{u}, \vec{v})=\int \vec{u}(x) \cdot \vec{v}(x) d x .
$$

In $\left(\dot{H}^{1}\left(\mathbb{T}^{d}\right)\right)^{d}$ we use the inner product notation

$$
((\vec{u}, \vec{v}))=\sum_{i=1}^{d}\left(D_{i} \vec{u}, D_{i} \vec{v}\right) .
$$

- Finally we introduce the trilinear expression

$$
b(\vec{u}, \vec{v}, \vec{w})=\int \vec{u}_{j} D_{j} \vec{v}_{i} \vec{w}_{i} d x=\int\langle\vec{u} \cdot \nabla \vec{v}, \vec{w}\rangle d x .
$$

Also we note that when $\vec{u}$ is divergence free, we have

$$
b(\vec{u}, \vec{v}, \vec{w})=\int\langle\nabla(\vec{v} \otimes \vec{u}), \vec{w}\rangle d x .
$$

We introduce the following definition:

Definition 4.7 Let $\vec{f} \in\left(H^{-\alpha}\left(\mathbb{T}^{d}\right)\right)^{d}, \alpha>0, \nabla \cdot \vec{f}=0$, and of mean zero ${ }^{9}$. A weak solution of the Navier-Stokes initial value problem (4.13) on $[0, T]$, is a function $\vec{u} \in L_{\text {loc }}^{2}((0, T) ; V) \cap$ $L_{\text {loc }}^{\infty}((0, T) ; H) \cap C_{\text {weak }}\left((0, T) ;\left(H^{-\alpha}\left(\mathbb{T}^{d}\right)\right)^{d}\right)$ satisfying $\frac{d \vec{u}}{d t} \in L_{\text {loc }}^{1}\left((0, T), V^{\prime}\right)$ and

$$
\begin{aligned}
& \left\langle\frac{d \vec{u}}{d t}, \vec{v}\right\rangle+((\vec{u}, \vec{v}))+b(\vec{u}, \vec{u}, \vec{v})=0 \quad \text { for a.e. } t \text { and for all } \vec{v} \in V, \\
& \lim _{t \rightarrow 0+} \vec{u}(t)=\vec{f} \quad \text { weakly in the }\left(H^{-\alpha}\left(\mathbb{T}^{d}\right)\right)^{d} \text { topology. }
\end{aligned}
$$

Theorem 4.8 (Existence and Uniqueness in 2D) Fix $T>0,0<\alpha<\frac{1}{2}$ and let $\vec{f} \in$ $\left(H^{-\alpha}\left(\mathbb{T}^{2}\right)\right)^{2}, \nabla \cdot \vec{f}=0$ and of mean zero. Then there exists a set $\Sigma \subset \Omega$ of probability 1 such that for any $\omega \in \Sigma$ the initial value problem (4.13) with datum $\vec{f}^{\omega}$ has a unique global weak solution $\vec{u}$ in the sense of Definition 4.7 of the form

$$
\vec{u}=\vec{u}_{\vec{f} \omega}+\vec{w}
$$

where $\vec{u}_{\vec{f} \omega}=e^{t \Delta} \overrightarrow{f^{\omega}}$ and $\vec{w} \in L^{\infty}\left([0, T] ;\left(L^{2}\left(\mathbb{T}^{2}\right)\right)^{2}\right) \cap L^{2}\left([0, T] ;\left(\dot{H}^{1}\left(\mathbb{T}^{2}\right)\right)^{2}\right)$.

Theorem 4.9 (Existence in 3D) Fix $T>0,0<\alpha<\frac{1}{4}$ and let $\vec{f} \in\left(H^{-\alpha}\left(\mathbb{T}^{3}\right)\right)^{3}, \nabla \cdot \vec{f}=0$, and of mean zero. Then there exists a set $\Sigma \subset \Omega$ of probability 1 such that for any $\omega \in \Sigma$ the initial value problem (4.13) with datum $\vec{f}^{\omega}$ has a global weak solution $\vec{u}$ in the sense of Definition 4.7 of the form

$$
\vec{u}=\vec{u}_{\vec{f} \omega}+\vec{w},
$$

where $\vec{u}_{\vec{f} \omega}=e^{t \Delta} \vec{f}^{\omega}$ and $\vec{w} \in L^{\infty}\left([0, T] ;\left(L^{2}\left(\mathbb{T}^{3}\right)\right)^{3}\right) \cap L^{2}\left([0, T] ;\left(\dot{H}^{1}\left(\mathbb{T}^{3}\right)\right)^{3}\right)$.

\footnotetext{
${ }^{9}$ This is assumed without loss of generality. Since the mean is conserved, if it is not zero, one can replace the solution with the solution minus the mean. This new function will satisfy an equation with a first order linear modification which does not effect the estimates.
} 
We now summarize the steps needed for the profs of the main Theorems 4.9 and 4.8.

Step 1: Set $\vec{u}_{\vec{f}^{\omega}}=e^{t \Delta} \vec{f}^{\omega}$. As we did in the previous section, instead of solving for $\vec{u}$ we solve for $\vec{w}$ where

$$
\vec{w}=\vec{u}-\vec{u}_{\vec{f} \omega},
$$

and hence $\vec{w}$ solves a difference equation of type

$$
\left\{\begin{array}{l}
\partial_{t} \vec{w}=\Delta \vec{w}-\mathbb{P} \nabla(\vec{w} \otimes \vec{w})+c_{1}[\mathbb{P} \nabla(\vec{w} \otimes \vec{g})+\mathbb{P} \nabla(\vec{g} \otimes \vec{w})]+c_{2} \mathbb{P} \nabla(\vec{g} \otimes \vec{g}) \\
\nabla \cdot \vec{w}=0, \\
\vec{w}(x, 0)=0,
\end{array}\right.
$$

where $\vec{g}=\vec{u}_{\vec{f} \omega}$.

Step 2: We show that in a set of almost full measure the function $\vec{u}_{\vec{f} \omega}$ satisfies certain estimates that are in fact not valid for the heat flow of a nonrandomized function in $H^{-\alpha}$. For example we have these estimates:

Proposition 4.10 Let $T>0$ and $\alpha \geq 0$. Let $r \geq p \geq q \geq 2, \sigma \geq 0$ and $\gamma \in \mathbb{R}$ be such that $(\sigma+\alpha-2 \gamma) q<2$. Then there exists $C_{T}>0$ such that for every $\vec{f} \in\left(H^{-\alpha}\left(\mathbb{T}^{d}\right)\right)^{d}$

$$
\left\|t^{\gamma}(-\Delta)^{\frac{\sigma}{2}} e^{t \Delta} \vec{f}^{\omega}\right\|_{L^{r}\left(\Omega ; L^{q}\left([0, T] ; L_{x}^{p}\right)\right.} \leq C_{T}\|\vec{f}\|_{H^{-\alpha}},
$$

where $C_{T}$ may depend also on $p, q, r, \sigma, \gamma$ and $\alpha$.

Moreover, if we set

$$
E_{\lambda, T, \vec{f}, \sigma, p}=\left\{\omega \in \Omega:\left\|t^{\gamma}(-\Delta)^{\frac{\sigma}{2}} e^{t \Delta} \vec{f}^{\omega}\right\|_{L^{q}\left([0, T] ; L_{x}^{p}\right)} \geq \lambda\right\},
$$

then there exists $c_{1}, c_{2}>0$ such that for every $\lambda>0$ and for every $\vec{f} \in\left(H^{-\alpha}\left(\mathbb{T}^{d}\right)\right)^{d}$

$$
P\left(E_{\lambda, T, \vec{f}, \sigma, p}\right) \leq c_{1} \exp \left[-c_{2} \frac{\lambda^{2}}{C_{T}\|\vec{f}\|_{H^{-\alpha}}^{2}}\right] .
$$

Proof For $t \neq 0$, using the notation $\vec{h}(x)=\langle-\Delta\rangle^{-\frac{\alpha}{2}} \vec{f}(x)$ and recalling the notation defined in (4.15) we have

$$
\begin{aligned}
t^{\gamma}(-\Delta)^{\frac{\sigma}{2}} e^{t \Delta} \vec{f}^{\omega}(x) & =t^{\gamma}(-\Delta)^{\frac{\sigma}{2}}\langle-\Delta\rangle^{\frac{\alpha}{2}} e^{t \Delta}\langle-\Delta\rangle^{-\frac{\alpha}{2}} \vec{f}^{\omega}(x) \\
& =t^{\gamma} \sum_{n \in \mathbb{Z}^{d}}|n|^{\sigma}\left(1+|n|^{2}\right)^{\frac{\alpha}{2}} e^{-t|n|^{2}} \widehat{\overrightarrow{h^{\omega}}}(n) e_{n}(x) \\
& \lesssim J_{\sigma}+J_{\sigma+\alpha}
\end{aligned}
$$

where for

$$
\beta \in\{\sigma, \sigma+\alpha\}
$$

we introduced $J_{\beta}$ as follows:

$$
J_{\beta}=t^{\gamma-\frac{\beta}{2}} \sum_{n \in \mathbb{Z}^{d}} t^{\frac{\beta}{2}}|n|^{\beta} e^{-t|n|^{2}} \widehat{\widehat{h^{\omega}}}(n) e_{n}(x) .
$$

In order to estimate $J_{\beta}$, we observe that $t^{\frac{\beta}{2}}|n|^{\beta} e^{-t|n|^{2}} \leq C$, which together with two applications of Minkowski's inequality, followed by a standard large deviation estimate, implies the 
first inequality in the following estimate:

$$
\begin{aligned}
\left\|J_{\beta}\right\|_{L^{r}\left(\Omega ; L^{q}\left([0, T] ; L_{x}^{p}\right)\right.} & \leq C_{r}\left\|\left(\sum_{n}\left|t^{\gamma-\frac{\beta}{2}} \widehat{\vec{h}}(n) e_{n}(x)\right|^{2}\right)^{\frac{1}{2}}\right\|_{L^{q}\left([0, T] ; L_{x}^{p}\right)} \\
& =C_{r}\left\|\sum_{n} t^{2 \gamma-\beta}\left|\widehat{\vec{h}}(n) e_{n}(x)\right|^{2}\right\|_{L^{\frac{q}{2}}\left([0, T] ; L_{x}^{\frac{p}{2}}\right)}^{\frac{1}{2}} \\
& \leq C_{r, p}\|\vec{h}\|_{L^{2}}\left(\int_{0}^{T}\left(\frac{1}{t^{\beta-2 \gamma}}\right)^{\frac{q}{2}}\right)^{\frac{1}{q}} \\
& =C_{r, p, q}\|\vec{h}\|_{L^{2}} T^{\frac{1}{q}+\frac{2 \gamma-\beta}{2}},
\end{aligned}
$$

as long as

$$
(\beta-2 \gamma) \frac{q}{2}<1
$$

which is for our range (4.31) of $\beta$ satisfied under the assumption that $(\sigma+\alpha-2 \gamma) q<2$. Now the estimate (4.27) follows from (4.30), (4.32) and (4.31).

To prove estimate (4.29) one uses the Bienaymé-Tchebishev's inequality as in Proposition 4.4 in [14].

Step 3: We show that if $\vec{g}$ satisfies certain estimates, such as for example those in Step 2 for $\vec{u}_{\vec{f} \omega}$ in an interval of time $[0, T]$, then the energy for the initial value problem (4.26)

$$
E(\vec{w})(t):=\|\vec{w}(t)\|_{L^{2}}^{2}+c \int_{0}^{t} \int_{\mathbb{T}^{d}}|\nabla \otimes \vec{w}|^{2} d x d s
$$

is bounded for all $t \in[0, T]$. To prove this step we need to use the Duhamel principle on (4.26) near $t=0$, and the classical energy estimates for the Navier-Stokes equation when $t$ is far from zero, this is Theorem 5.1 in [55]. The tricky part in implementing these estimates at these two regimes is that one has to first show that the solutions obtained via the Duhamel principle and weak solutions are equivalent, at least under certain conditions for $\vec{g}$. This is the content of Lemma 4.2 in [55].

Step 4: Once one proves the boundedness for the energy of $\vec{w}$, assuming certain conditions on $\vec{g}$, a Galerkin scheme is implemented and a weak solution is obtained on an arbitrary interval of time. This is Theorem 6.1 in [55].

Step 5: One then defines the set

$$
E_{j}:=\left\{\omega \in \Omega /\left\|\vec{u}_{\vec{f} \omega}\right\|_{*}>2^{j}\right\}
$$

where $\|\cdot\|_{*}$ contains all the norms that need to be bounded for $\vec{u}_{\vec{f} \omega}$ in order to implement the steps above for the difference equation (4.26) with $\vec{g}=\vec{u}_{\vec{f} \omega}$. Large deviation estimates such as the one in Proposition 4.10 gives that

$$
P\left(E_{j}\right) \leq c_{1} \exp \left[-c_{2} \frac{2^{2 j}}{C_{T}\|\vec{f}\|_{H^{-\alpha}}^{2}}\right]
$$

hence if $\Sigma=\cap E_{j}^{c}$ it follows that $P(\Sigma)=1$ and the conclusion ${ }^{10}$ of Theorems 4.8 and 4.8 hold.

\footnotetext{
${ }^{10}$ What we described is the procedure to obtain global weak solutions, for the uniqueness result in Theorem 4.8 see Theorem 7.1 in [55].
} 


\section{Global flows for random surface quasi-geostrophic equations}

In this section we consider the surface quasi-geostrophic equation SQG where randomness is injected into the system via the data or via a random diffusion term. We discuss the work in [56] about the invariance of the associated Gaussian measure and global existence of weak solutions in the first case, and the work in [11] exhibiting a 'regularization by noise' effect and proving the existence of pathwise unique global solutions in the second.

5.1 Invariant measure and longtime weak solutions to the inviscid modified SQG equation

In this section we summarize the results in [56] where an inviscid modified surface quasi geostrophic equation $\mathrm{mSQG}$ is considered and randomness is injected into the system via the data. We prove invariance of the associated Gibbs measure and global existence of weak solutions.

The mSQG equation is also an active scalar equation describing the transport of the scalar valued function $\theta=\theta(x, t): \mathbb{T}^{2} \times[0, \infty) \rightarrow \mathbb{R}$ under the velocity field $u$, which itself is related to $\theta$ now via a regularized Biot-Savart law. More precisely, the mSQG equation that we consider in this paper is given as follows:

$$
\left\{\begin{array}{l}
\theta_{t}+(u \cdot \nabla) \theta=0, \quad x \in \mathbb{T}^{2} \text { and } t>0 \\
u=\mathcal{R}^{\perp}|D|^{-\delta} \theta,
\end{array}\right.
$$

where $\delta>0$, and $\mathcal{R}^{\perp}:=\nabla^{\perp}(-\Delta)^{-1 / 2}=\nabla^{\perp}|D|^{-1}$ denotes the Riesz transform. Here $\nabla^{\perp}=$ $\left(-\partial_{x_{2}}, \partial_{x_{1}}\right)$.

When $\delta=1$ the equation (5.1) coincides with the 2D Euler equation with $\theta$ representing the vorticity $\omega$. When $\delta=0$ the equation (5.1) coincides with the inviscid surface quasi geostrophic SQG equation. For $0<\delta<1$ the relation between the velocity $u$ and the function $\theta$ is less singular than in the case of the SQG equation, but more singular than in the case of the $2 \mathrm{D}$ Euler equation, see [56]. Note that the inviscid SQG equation is one derivative less regular than the $2 D$ Euler equation.

The paper [56] we use probabilistic tools to obtain a global flow in $H^{s}\left(\mathbb{R}^{2}\right), s<-3+\delta$, for any $0<\delta \leq 1$, for the mSQG equation (5.1) in the support of a Gaussian measure $\rho$, which is left invariant under the flow. In particular, the approach of [1] in the context of mSQG is implemented as follows:

1. We work with the streamline formulation of the equation, whose (sufficiently) smooth solutions still conserve 'enstrophy' (see below). Then we consider the infinite Gaussian measure $\rho$ constructed with respect to this enstrophy.

2. We rewrite the streamline formulation of the equation in terms of an orthonormal $L^{2}$ basis as an infinite ODE system, for which we analyze the coefficient corresponding to the nonlinear term, with the goal of obtaining an expectation estimate that will allow subsequent probabilistic analysis. For this part we will give more details below.

3. We introduce an approximate system of ODE, which is still an infinite system, but which has truncated nonlinear term. We show that each of these systems has a global flow and leaves the Gaussian measure $\rho$ invariant. 
4. Finally we perform a probabilistic convergence argument, which gives a global flow for the streamline formulation of the equation in the support of the Gaussian measure $\rho$.

To state the result precisely we need to introduce some notation. First we introduce the streamline function $\varphi$ for our equation (5.1) so that $u=\nabla^{\perp} \varphi$. Such streamline function $\varphi$ is related to $\theta$ via $\varphi=|D|^{-1-\delta} \theta$. We find it convenient to rewrite the streamline formulation in terms of the regularized stream function $\psi$ introduced via

$$
|D|^{\delta} \varphi=\psi
$$

Then the regularized streamline formulation that we work with reads as follows:

$$
\left\{\begin{array}{l}
\psi_{t}+|D|^{-1}(u \cdot \nabla)|D| \psi=0 \\
u=\nabla^{\perp}|D|^{-\delta} \psi
\end{array}\right.
$$

Below we will abbreviate the nonlinearity in $(5.3)$ by

$$
B(\psi, \psi):=-|D|^{-1}\left(\nabla^{\perp}|D|^{-\delta} \psi \cdot \nabla\right)|D| \psi
$$

We recall that for classical solutions to (5.1) the SQG enstrophy $\|\theta\|_{L^{2}}$ is conserved in time. We also note that, thanks to (5.2), we have that $\psi$ is mean zero. Consequently, the homogeneous and inhomogeneous Sobolev spaces restricted to our space of solutions coincide. We therefore take the Sobolev norm

$$
\|f\|_{H^{s}\left(\mathbb{T}^{2}\right)}^{2}:=\sum_{k \in \mathbb{Z}^{2}, k \neq 0}|k|^{2 s}|\hat{f}(k)|^{2},
$$

and we introduce the spaces

$$
X^{\sigma}:=\bigcap_{s<\sigma} H^{s} .
$$

Similarly, we slightly abuse notation by defining the associated path space by

$$
C\left([0, T]: X^{\sigma}\right):=\bigcap_{s<\sigma} C\left([0, T]: H^{s}\right)
$$

Since both of these spaces are defined as nested intersections, they inherit the natural subspace topology.

Finally, it follows from the definitions of $\psi$ and $\varphi$ and the conservation of $\|\theta\|_{L^{2}}$ for solutions $\theta$ of (5.1) that $\|\psi\|_{H^{1}}$ is formally conserved in time. It is this conservation of $\|\psi\|_{H^{1}}$ that gives rise to the Gaussian measure $\rho$ that we informally write as:

$$
\text { " } d \rho(\psi):=\frac{1}{Z} \prod_{k \in \mathbb{Z}^{2}, k \neq 0} \exp \left(-2|k|^{2}\left|\psi_{k}\right|^{2}\right) d \psi_{k} "
$$

where $d \psi_{k}:=d x_{k} d y_{k}$ is the Lebesgue measure on $\mathbb{C}$ associated to the variable $\psi_{k}=x_{k}+$ $i y_{k} \in \mathbb{C}$, and $Z$ is the appropriate normalization factor needed to yield a probability measure. Unfortunately this heuristic expression is not well-defined, but it can be made meaningful [7]. With the construction of the Gaussian measure $\rho$ one can state the result that establishes global flows for the regularized streamline formulation (5.3) of the mSQG equation. More precisely: 
Theorem 5.1 Let $T>0$ be given. Then there exists a flow $\tilde{\Psi}(\omega, t)$ defined on a probability space $(\tilde{\Omega}, \tilde{\mathcal{F}}, \tilde{P})$ with values in $C\left([0, T]: X^{-2}\right)$ such that for $\tilde{P}$-almost every $\omega \in \tilde{\Omega}$,

$$
\tilde{\Psi}(\omega, t)=\tilde{\Psi}(0, \omega)+\int_{0}^{t} B(\tilde{\Psi}(\omega, \tau)) d \tau
$$

where $B$ is as in (5.4), as well as a Gaussian measure $\rho$ supported on $X^{-2}$ which is invariant with respect to $\tilde{\Psi}(t, \omega)$, i.e., for all measurable $F: X^{-2} \rightarrow \mathbb{R}$ and $t \in[0, T]$,

$$
\int_{\tilde{\Omega}} F(\tilde{\Psi}(\omega, t)) d \tilde{P}(\omega)=\int_{X^{-2}} F(\psi) d \rho(\psi)
$$

In this survey we decided to give details only on the expansion of (5.3) explicitly into an infinite system of ODEs in the Fourier frequencies, and on showing that the $H^{s}$-norm of the nonlinearity of the equation is finite in $L_{\rho}^{2}$ provided that $s<-2$. Informally, this calculation shows that $X^{-2}$ is the smallest space with respect to which the system (5.3) is closed in $\rho$ expectation. Moreover this estimate is a key ingredient of the compactness argument used to construct the random flows and to obtain the main result. We will only outline the steps of the proof of the main result in Theorem 5.1

5.1.1 An infinite system of ODE and the expectation of its nonlinear term

Write $\psi=\sum_{k \in \mathbb{Z}^{2}} \psi_{k} e_{k}$, where $\left\{e_{k}\right\}$ is an orthonormal basis of $L^{2}\left(\mathbb{T}^{2}\right)$, and rewrite the modified streamline formulation (5.3) in terms of coefficients with respect to this orthonormal basis as follows:

$$
\frac{d \psi_{k}}{d t}=B_{k}(\psi, \psi)
$$

where $B_{k}$ denotes the coefficients of the nonlinearity $B$

$$
B(\psi, \psi):=-|D|^{-1}\left(\nabla^{\perp}|D|^{-\delta} \psi \cdot \nabla\right)|D| \psi
$$

in this basis.

We calculate the coefficients $B_{k}$ for $k \neq 0$ to be

$$
\begin{aligned}
B_{k} & =\sum_{h+h^{\prime}=k, h, h^{\prime} \neq 0}-|k|^{-1}|h|^{-\delta} h^{\perp} \cdot h^{\prime}\left|h^{\prime}\right| \psi_{h} \psi_{h^{\prime}} \\
& =\frac{1}{2}\left(\sum_{h+h^{\prime}=k, h, h^{\prime} \neq 0}|k|^{-1}\left|h^{\prime}\right|^{-\delta} h^{\perp} \cdot h^{\prime}|h| \psi_{h} \psi_{h^{\prime}}+\sum_{h+h^{\prime}=k, h, h^{\prime} \neq 0}-|k|^{-1}|h|^{-\delta} h^{\perp} \cdot h^{\prime}\left|h^{\prime}\right| \psi_{h} \psi_{h^{\prime}}\right) \\
& =\frac{1}{2} \sum_{h+h^{\prime}=k, h, h^{\prime} \neq 0}|k|^{-1}\left(h^{\perp} \cdot h^{\prime}\right)\left(\left|h^{\prime}\right|^{-\delta}|h|-|h|^{-\delta}\left|h^{\prime}\right|\right) \psi_{h} \psi_{h^{\prime}},
\end{aligned}
$$

where in the above we symmetrized the sum using the divergence-free structure with an eye towards minimizing the number of positive factors of $|h|$ and $\left|h^{\prime}\right|$. This gives

$$
B_{k}=-\frac{1}{2} \sum_{h \neq 0, k}\left(h^{\perp} \cdot \frac{k}{|k|}\right)\left(|k-h|^{-\delta}|h|-|h|^{-\delta}|k-h|\right) \psi_{h} \psi_{k-h}=: \sum_{h \neq 0, k} \alpha_{k, h} \psi_{h} \psi_{k-h} .
$$

Notice that one can readily check that $\alpha_{k, h}=\alpha_{k, k-h}$ for all $k, h \in \mathbb{Z}^{2}$.

The subsequent analysis depends strongly on controlling the expectation of the nonlinearity in a rough Sobolev space. We then have the following crucial proposition:

Proposition 5.2 Let $0<\delta \leq 1$. Then $B$ given by (5.8) satisfies

$$
B \in L_{\rho}^{2}\left(H^{s}, H^{s}\right), \quad \text { for all } s<-2 .
$$


Proof We need to show that expectation of the expression $\|B(\psi)\|_{H^{s}}^{2}$ is finite. Using the expectations of the moments as well as the fact that $\alpha_{k, h}=\alpha_{k, k-h}$, we first have that

$$
\begin{aligned}
\mathbb{E}_{\rho}\left(\|B\|_{H^{s}}^{2}\right) & =\sum_{k \neq 0}|k|^{2 s} \sum_{h, h^{\prime} \neq 0} \alpha_{k, h} \alpha_{k, h^{\prime}} \mathbb{E}_{\rho}\left(\psi_{h} \psi_{k-h} \bar{\psi}_{h^{\prime}} \bar{\psi}_{k-h^{\prime}}\right) \\
& =2 \sum_{k \neq 0}|k|^{2 s} \sum_{h, h^{\prime} \neq 0} \frac{\alpha_{k, h} \alpha_{k, h^{\prime}}}{|h|^{2}|h-k|^{2}}\left(\delta_{h, h^{\prime}}+\delta_{h, k-h^{\prime}}\right) \\
& =4 \sum_{k \neq 0}|k|^{2 s} \sum_{h, h^{\prime} \neq 0} \frac{\alpha_{k, h}^{2}}{|h|^{2}|h-k|^{2}} .
\end{aligned}
$$

We focus first on establishing that the inner sum in this last expression converges ${ }^{11}$. Substituting our expression for $\alpha_{k, h}$ in (5.9) we have

$$
\begin{aligned}
\sum_{h \neq 0, k} \frac{\alpha_{k, h}^{2}}{|h|^{2}|h-k|^{2}} & \lesssim \sum_{h \neq 0, k} \frac{\left(|k-h|^{-\delta}|h|-|h|^{-\delta}|k-h|\right)^{2}}{|h-k|^{2}} \\
& \lesssim \sum_{h \neq 0, k} \frac{|h-k|^{-2 \delta}(|h|-|k-h|)^{2}}{|h-k|^{2}}+\sum_{h \neq 0, k} \frac{\left(|h|^{-\delta}-|h-k|^{-\delta}\right)^{2}(|h|-|k-h|)^{2}}{|h-k|^{2}} \\
& +\sum_{h \neq 0, k} \frac{|h|^{2}\left(|k-h|^{-\delta}-|h|^{-\delta}\right)^{2}}{|h-k|^{2}} \\
& :=S_{1}+S_{2}+S_{3} .
\end{aligned}
$$

In estimates on $S_{1}$ and $S_{2}$ we utilize the immediate consequence of the triangle inequality:

$$
|| h|-| k-h|| \leq|k| .
$$

Then, for any $\delta>0$, the sum $S_{1}$ can be bounded from above as follows:

$$
S_{1} \leq \sum_{h \neq 0, k} \frac{|k|^{2}}{|k-h|^{2+2 \delta}} \lesssim|k|^{2} .
$$

Next, utilizing (5.11) and decomposing $S_{2}=S_{2}^{l o}+S_{2}^{h i}$ depending on whether $|h|$ is less that or greater than $2|k|$ respectively, and using Lemma 4.1 in [56] we have that

$$
\begin{aligned}
S_{2} & \lesssim \sum_{\substack{|h| \leq 2|k|, h \neq 0, k\\
}} \frac{|k|^{2}}{|h|^{2 \delta}|k-h|^{2}}+\sum_{|h| \leq 2|k| h \neq 0, k} \frac{|k|^{2}}{|k-h|^{2+2 \delta}}+\sum_{|h| \geq 2|k|, k \neq 0} \frac{\delta^{2}|k|^{4}|h|^{-2-2 \delta}}{|h-k|^{2}} \\
& \lesssim|k|^{2}+|k|^{2-2 \delta} .
\end{aligned}
$$

Decomposing $S_{3}$ similarly into 'high-low' and again applyingLemma 4.1 in [56], we have:

$$
\begin{aligned}
S_{3} & \lesssim \sum_{\substack{|h| \leq 2|k|, h \neq 0, k\\
}} \frac{|h|^{2}}{|h|^{2 \delta}|k-h|^{2}}+\sum_{|h| \leq 2|k| h \neq 0, k} \frac{|h|^{2}}{|k-h|^{2+2 \delta}}+\sum_{|h| \geq 2|k|, k \neq 0} \frac{|h|^{2} \delta^{2}|k|^{2}|h|^{-2-2 \delta}}{|h-k|^{2}} \\
& \lesssim|k|^{2}+|k|^{2-2 \delta} .
\end{aligned}
$$

The maximum amount of smoothness imposed on $k$ from evaluating these sums is comparable to $|k|^{2}$. Therefore, the expectation at the beginning of the calculation can be estimated by

$$
\mathbb{E}_{\rho}\left(\|B\|_{H^{s}}^{2}\right) \lesssim \sum_{k}|k|^{2 s+2},
$$

\footnotetext{
${ }^{11}$ This is precisely the step that fails in the classical inviscid SQG model with $\delta=0$.
} 
which is finite provided we choose $s<-2$.

5.1.2 Outline of the proof of Theorem 5.1

As we did in the previous sections we indicate below the main steps of the proof.

Step 1: We introduce the $N$ th approximate flow $\Psi^{N}(t, \psi)$ as the solution of the Cauchy problem

$$
\left\{\begin{array}{l}
\partial_{t} \Psi^{N}(t)=B^{N}\left(\Psi^{N}(t), \Psi^{N}(t)\right) \\
\Psi^{N}(0, \psi)=\psi
\end{array}\right.
$$

If we let $V^{N}$ satisfy the finite dimensional system

$$
\left\{\begin{array}{l}
\partial_{t} V^{N}(t)=B^{N}\left(V^{N}, V^{N}\right) \\
V^{N}(0, \psi)=\Pi_{N} \psi,
\end{array}\right.
$$

then the flow $\Psi^{N}$ can be decomposed into

$$
\Psi^{N}(t, \psi)=V^{N}\left(t, \Pi_{N} \psi\right)+\Pi_{N}^{\perp} \psi
$$

that is, the dynamics of the approximate flows are only nontrivial on finite dimensional subspaces. The $N$-th approximate $\Psi^{N}$ still conserve $H^{1}$ and are divergence free. If we denote the

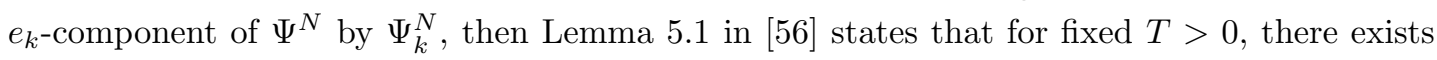
a unique flow $\Psi^{N}(t, \psi)$ solving (5.13) for all $t \in[0, T]$ with $\Psi_{k}^{N}(t, \psi) \in C([0, T], \mathbb{C})$ and which leaves the measure $\rho$ invariant.

Step 2: In order to construct random flows from the (essentially) finite-dimensional deterministic flows, we regard the deterministic flows $\Psi^{N}(t, \psi)$ as stochastic processes sampled from $X^{-2}$ with state space $C\left([0, T]: X^{-2}\right)$, and introduce the measures $\nu_{N}$ supported on the infinite dimensional path space $C\left([0, T]: X^{-2}\right)$ as their laws:

$$
\nu_{N}(\Gamma)=\rho\left(\left\{\psi \in X^{-2}: \Psi^{N}(\psi, \cdot) \in \Gamma\right\}\right), \quad \Gamma \subset C\left([0, T]: X^{-2}\right) .
$$

Our first goal is to show that the laws $\nu_{N}$ can be used to construct a measure $\nu$ supported on $C\left([0, T]: X^{-2}\right)$ that will serve as the law of our eventual candidate flows. This follows from two fundamental tools, a compactness argument and Skorokhod Lemma, as follows.

Prokhorov Lemma states that tightness of $\left(\nu^{(N)}\right)$ implies $\left(\nu^{(N)}\right)$ is weakly sequentially compact $^{12}$. Tightness means that for each $\epsilon>0$, there is a compact subset $K_{\epsilon} \subset C\left([0, T]: X^{-2}\right)$ so that $\nu^{(N)}\left(K_{\epsilon}\right) \geq 1-\epsilon$ uniformly in $N$.

In [56] we prove that for $T>0$ and any $s<-2$ the family $\left\{\nu^{(N)}\right\}$ of measures is tight in $C\left([0, T]: H^{s}\right)$ which is obtained by showing that solutions lie in a Hölder-continuous subspace of $C\left([0, T]: H^{s}\right)$ that is pre-compact by Sobolev embedding. The proof of tightness crucially uses invariance, $\psi_{0} \in X^{-2}$ and $\mathbb{E}_{\rho}\left(\|B\|_{H^{s}}^{2}\right)$ is finite for $s<-2$. Then for any fixed $s<-2$, Prokhorov implies the existence of a subsequence of measures which converges weakly to another measure $\nu_{s}$. A standard diagonalization argument allows one then to extract a weak limiting measure $\nu$ on $C\left([0, T]: X^{-2}\right)$.

Now, Skorokhod Lemma ensures that there is a probability space $(\tilde{\Omega}, \tilde{\mathcal{F}}, \tilde{P})$ and random processes $\tilde{\Psi}^{(N)}(\omega), \tilde{\Psi}(\omega)$ with values in $C\left([0, T]: X^{-2}\right)$ so that:

\footnotetext{
${ }^{12}$ i.e. can extract a weakly convergent subsequence.
} 
1. $\tilde{\Psi}^{(N)}(\omega) \rightarrow \tilde{\Psi}(\omega)$ in $C\left([0, T]: X^{-2}\right) \quad$ P-a.s. in $\omega$

2. $\nu$ is the law of $\tilde{\Psi}$, i.e.,

$$
\nu(\Gamma)=P(\{\omega \in \tilde{\Omega}: \tilde{\Psi}(\omega, t) \in \Gamma\}) \quad \Gamma \subset C\left([0, T]: X^{-2}\right)
$$

3. Each $\nu^{(N)}$ is the law of $\tilde{\Psi}^{(N)}$.

This $\tilde{\Psi}$ is our candidate for the random flow. Note that by construction the laws of $\tilde{\Psi}^{(N)}$ and that of $\Psi^{(N)}$ in the path space $C\left([0, T]: X^{-2}\right)$ are the same.

To conclude the argument we verify that $\rho$ is an invariant measure for the (random) flow $\tilde{\Psi}$ from Skorokhod. In particular we show that $\forall F: X^{-2} \rightarrow \mathbb{R}$ measurable and $t \in[0, T]$ :

$$
\int_{\Omega} F(\tilde{\Psi}(\omega, t)) d \tilde{P}(\omega)=\int_{X^{-2}} F(\psi) d \rho(\psi) .
$$

This in turn implies that $\tilde{\Psi}$ takes values in $X^{-2}$ a.s. in $\tilde{\Omega}$. We also have the following almost everywhere pointwise equation for $\tilde{\Psi}^{N}$ :

$$
\tilde{\Psi}^{N}(t, \omega)=\tilde{\Psi}^{N}(0, \omega)+\int_{0}^{t} B^{N}\left(\tilde{\Psi}^{N}(\tau, \omega)\right) d \tau, \quad \text { a.e. } \omega \in \tilde{\Omega} .
$$

Finally we take the limit $N \rightarrow \infty$ in the truncated equations to show that $\tilde{\Psi}$ satisfies the equation $P$-almost surely. By the construction using Skorokhod's Lemma, we already have that $\tilde{\Psi}^{N}(t, \omega) \rightarrow \tilde{\Psi}(t, \omega)$ and $\tilde{\Psi}^{N}(0, \omega) \rightarrow \tilde{\Psi}(0, \omega)$ for each $t \in[0, T]$, a.s. in $\tilde{\Omega}$. Hence to show $P$-a.e. convergence of the Duhamel term it suffices to show (possibly up to the extraction of another subsequence) that as $N \rightarrow \infty$,

$$
\int_{\tilde{\Omega}} \mid \int_{0}^{t} B^{N}\left(\tilde{\Psi}^{N}(\omega, \tau) d \tau-\int_{0}^{t} B(\tilde{\Psi}(\omega, \tau)) d \tau \mid d \tilde{P}(\omega) \rightarrow 0 .\right.
$$

We refer the reader to [56] for full details.

One may ask about uniqueness; this question is hard. Known deterministic arguments are not applicable due to the low regularity of the flow. Furthermore, the criteria in the work of Ambrosio-Figalli [2] is not applicable either since the nonlinearity does not map into $H^{1}$, the Cameron-Martin space.

5.2 Unique global smooth solutions for the inviscid SQG equation perturbed by random diffusion

In this section we describe the results obtained in [11] where we consider the surface quasigeostrophic equation SQG where randomness is injected into the system via a random diffusion term behaving like white noise in time. We discuss the effect of 'regularization by noise', that is noise improving the behavior over a purely deterministic setting [30], and show the existence of pathwise unique global smooth solutions.

Consider the SQG equation for $\theta: \mathbb{R} \times \mathbb{T}^{2} \rightarrow \mathbb{R}$ with a random in time diffusion, written as an Itô stochastic integral:

$$
d \theta+R^{\perp} \theta \cdot \nabla \theta d t=\nu|\nabla|^{s} \theta d W_{t}, \quad \theta(0)=\theta_{0},
$$


where $R^{\perp}=\left(-R_{2}, R_{1}\right)$ and $W_{t}$ is a standard Wiener process in time. The index $0<s \leq 1$ and the constant of diffusion $\nu>0$ are fixed.

Let $A:=|\nabla|^{2 s}$ and let $\phi(t)$ be suitably chosen to be of the form:

$$
\phi(t)=\alpha+\beta t \quad \text { with } \alpha>0 \text { and } 0<\beta<\frac{\nu^{2}}{2},
$$

or shifts $\phi\left(t-T_{0}\right)$ of it, where $T_{0}$ is fixed.

We work with $L^{2}$-weighted norms by $A^{m} e^{\phi(t) A^{1 / 2}}$; that is on Gevrey-type spaces defined precisely below. First, recall $v \in C^{\infty}\left(\mathbb{T}^{2}\right)$ is in the Gevrey class $\eta>0$ if there exists $M>$ $0, \tau>0$ such that

$$
\left|\partial^{\beta} v(x)\right| \leq M \frac{(\beta !)^{\eta}}{\tau^{|\beta|}} \quad \text { for all } \beta, x .
$$

We work with an equivalent characterization given by Foias and Temam in [31] and which we can state as follows. First, let $\gamma>0$ and $\varphi$ be a continuous real function. Then $w$ : $[0, T] \times \mathbb{T}^{2} \rightarrow \mathbb{R}$

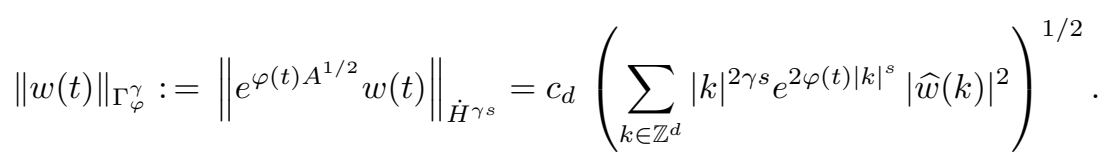

Define then the corresponding Gevrey space as

$$
\Gamma_{\varphi}^{\gamma}:=\left\{f \in \dot{H}^{\gamma s}:\|f\|_{\Gamma_{\varphi}^{\gamma}}<\infty\right\} .
$$

From this, one obtains the combined space-time spaces

$$
C_{T}^{0} \Gamma_{\varphi}^{\gamma}:=\left\{f \in C^{0}\left([0, T], \Gamma_{\varphi}^{\gamma}\right):\|f\|_{C_{T}^{0} \Gamma_{\varphi}^{\gamma}}<\infty\right\} .
$$

In [11] we prove the following theorem.

Theorem 5.3 (Main Theorem) Let $\frac{1}{s}<\sigma<2$ and $\theta_{0} \in \dot{H}^{\sigma s}$ be given with $\widehat{\theta}_{0}(0)=0$ and

$$
\left\|e^{\alpha A^{1 / 2}} \theta_{0}\right\|_{\dot{H}^{\sigma s}}=: E<\frac{\nu^{2}}{2}
$$

for some $\alpha>0$. Then there exists $\beta=\beta(\nu, E)<E$ such that for $0<\epsilon<\alpha$ we obtain a pathwise unique, global solution $\theta \in C^{0} \dot{H}^{\sigma s}$ with probability

$$
P(\alpha, \epsilon, \nu, E) \geq 1-e^{-2 \frac{(\alpha-\epsilon) \beta}{\nu^{2}}} .
$$

In particular, for any $p_{0} \in(0,1)$ and $E>0$ given, there exist $\nu>0$ and $\alpha>0$ such that if the initial data $\theta_{0}$ satisfy (5.19) for these values we obtain a path-wise unique global solution with likelihood at least $p_{0}$.

A key idea to prove the theorem is to transform the equation into a pathwise deterministic equation; that is an equation for which for each instance of the Brownian motion $t \rightarrow W_{t}(\omega)$ we have a deterministic equation with diffusion. Such transformation is motivated by the use of the geometric Brownian motion in [32]. More precisely, if we set

$$
\Gamma(t):=e^{-\nu|\nabla|^{s} W_{t}}
$$

then, by Itô calculus, we have that

$$
d \Gamma=-\nu|\nabla|^{s} \Gamma d W_{t}+\frac{1}{2} \nu^{2}|\nabla|^{2 s} \Gamma d t,
$$


so for $u(t):=\Gamma(t) \theta(t)$

$$
d u=-\Gamma\left(R^{\perp} \Gamma^{-1} u \cdot \nabla \Gamma^{-1} u\right) d t-\frac{1}{2} \nu^{2}|\nabla|^{2 s} u d t,
$$

and hence

$$
\left\{\begin{array}{l}
\partial_{t} u+\Gamma\left(R^{\perp} \Gamma^{-1} u \cdot \nabla \Gamma^{-1} u\right)=-\frac{1}{2} \nu^{2}|\nabla|^{2 s} u, \\
u(0)=u_{0}=\theta_{0} .
\end{array}\right.
$$

Remark 5.4 If we denote by

$$
B(f, g):=\Gamma\left(R^{\perp} \Gamma^{-1} f \cdot \nabla \Gamma^{-1} g\right),
$$

and we normalize by switching to the variable $v:=|\nabla|^{\sigma s} e^{\phi(t) A^{1 / 2}} u$, so that

$$
\hat{u}(t, j)=e^{-\phi(t)|j|^{s}}|j|^{-\sigma s} \hat{v}(t, j),
$$

and $\|\hat{v}(t, j)\|_{\ell_{j}^{2}}=\|v(t)\|_{L^{2}}=\left\|e^{\phi(t) A^{1 / 2}} u(t)\right\|_{\dot{H}^{\sigma s}}$, then the nonlinearity reads

$$
\widehat{B(u, u)}(t, k)=\sum_{j \in \mathbb{Z}^{2} \backslash\{0\}} \frac{(k-j) \cdot j^{\perp}}{|k-j|^{\sigma s}|j|^{1+\sigma s}} e^{-\nu W_{t}\left[|k|^{s}-|k-j|^{s}-|j|^{s}\right]} e^{\phi(t)\left[-|k-j|^{s}-|j|^{s}\right]} \hat{v}(k-j) \hat{v}(j) .
$$

Our strategy entails establishing local well-posedness for the transformed initial value problem (5.20), and prove energy estimates in the Gevrey spaces introduced above. Armed with these we then proceed to show global existence of solutions in this pathwise deterministic setting by an iteration that carefully intertwines the local theory with the energy estimates and relies on the following probability estimate for Brownian motion:

For $\alpha, \beta>0$, the probability that the process $W(t)-\beta t>\alpha$ in finite time is given by $e^{-2 \alpha \beta}$.

This implies that for our specific choice of $\phi(t)=\alpha+\beta t$ above - for suitable $\beta \sim \nu^{2}$ (diffusion constant)-, the condition $\phi(t)-\nu W_{t} \geq 0$ holds globally on an exponentially large set. Finally, at the very end, we come back to the original equation, to obtain our main theorem.

Let us start with our local well-posedness result for (5.20).

Proposition 5.5 Let $T_{0}>0$ and $I \subset \mathbb{R}$ be an open interval with $T_{0} \in I$. Assume $\phi(t)-\nu W_{t} \geq$ 0 for $t \in I$, and $\phi\left(T_{0}\right)=\alpha$.

Fix $\sigma>0$ with $\frac{1}{s}<\sigma<2$. Then for initial data $u_{0}$ with $e^{\phi\left(T_{0}\right) A^{1 / 2}} u_{0} \in \dot{H}^{\sigma s}$ (and $\hat{u}_{0}(0)=0^{13}$ ) there exist $T>0$, depending only on the norm of the initial data, and a unique solution $u \in C^{0}\left(I \cap\left[T_{0}, T_{0}+T\right], \Gamma_{\phi}^{\sigma}\right)$ of $(5.20)$.

Remark 5.6 The condition that $s \leq 1$ is crucial in our proof of Proposition 5.5 to absorb the propagators $e^{-\nu W_{t}|\nabla|^{s}}$ using the exponential weights of our Gevrey spaces. We also use that $\phi(t)-\phi(\tau)=\beta(t-\tau)$ and $\beta<\frac{\nu^{2}}{2}$.

On the other hand, given $\phi(t)-\nu W_{t} \geq 0$ for all $t \in\left[T_{0}, T\right]$, the following bilinear bounds on $\left[T_{0}, T\right]$ easily follow:

$$
\left|\left\langle e^{\phi(t) A^{1 / 2}} B(w, w), e^{\phi(t) A^{1 / 2}} A w\right\rangle_{L^{2}}\right| \lesssim\left\|e^{\phi(t) A^{1 / 2}} w\right\|_{\dot{H}^{\sigma s}}\left\|e^{\phi(t) A^{1 / 2}} w\right\|_{\dot{H}^{(\sigma+1) s}}^{2} .
$$

Relying on (5.21) we can then establish the necessary energy estimates:

\footnotetext{
${ }^{13}$ Note that $\int u d x=$ constant so WLOG can assume mean zero.
} 
Proposition 5.7 Assume $\sigma \in\left(\frac{1}{s}, 2\right)$ and let $u$ be a local solution on $\left[T_{0}, T\right]$ of (5.20) that is bounded in $C^{0}\left(\left[T_{0}, T\right], \Gamma_{\phi}^{\sigma+1}\right)$. Assuming that $\phi(t)-\nu W_{t} \geq 0$ and that

$$
\left\|e^{\phi\left(T_{0}\right) A^{1 / 2}} u\left(T_{0}\right)\right\|_{\dot{H}^{\sigma s}} \leq \frac{\nu^{2}}{2}-\beta
$$

for $t \in\left[T_{0}, T\right]$ we have

$$
\left\|e^{\phi(t) A^{1 / 2}} u(t)\right\|_{\dot{H}^{\sigma s}} \leq\left\|e^{\phi\left(T_{0}\right) A^{1 / 2}} u\left(T_{0}\right)\right\|_{\dot{H}^{\sigma s}} .
$$

Armed with propositions 5.5 and 5.7 we can then show the existence of pathwise unique global solutions to equation (5.20). Namely in [11] we show that for $\sigma \in\left(\frac{1}{s}, 2\right)$, and initial data $u_{0}$ satisfying

$$
\left\|u_{0}\right\|_{\Gamma_{\alpha+\epsilon}^{\sigma}}=\left\|e^{(\alpha+\epsilon) A^{1 / 2}} u_{0}\right\|_{\dot{H}^{\sigma s}} \leq \frac{\nu^{2}}{2}-\beta
$$

for some $\alpha>0, \epsilon>0$ and $0<\beta<\frac{\nu^{2}}{2}$, if we let $\phi(t):=\alpha+\beta t$, then with probability at least $1-e^{-2 \frac{\alpha \beta}{\nu^{2}}}$ there exists a pathwise ${ }^{14}$ unique global solution $u \in C^{0}\left([0, \infty), \Gamma_{\phi}^{\sigma}\right)$ to $(5.20)$. Moreover, the mapping

$$
t \mapsto\|u(t)\|_{\Gamma_{\phi}^{\sigma}}=\left\|e^{\phi(t) A^{1 / 2}} u(t)\right\|_{\dot{H}^{\sigma s}}
$$

is pathwise monotonically decreasing.

In short, we show that for any size $E$ of initial data and any probability $p \in[0,1)$, for a sufficiently large parameter $\nu$ and sufficiently smooth initial data (i.e. $\alpha$ sufficiently large) we obtain global solutions with probability at least $p$.

The energy estimates above require finiteness of a higher norm than the one for which they give monotonicity, which means we have to control two levels of regularity; ie. in order to show that the $\Gamma_{\phi}^{\sigma}$ norm is monotonically decreasing on a given time interval, we have to guarantee that the $\Gamma_{\phi}^{\sigma+1}$ norm remains finite on that same interval. But this cannot be done using just Proposition 5.5 since a priori the higher energy norm will only be controlled for a shorter amount of time. The key point here is that - thanks to our Gevrey setting - we can achieve what we need by just an $\epsilon$ loss in regularity: $\|v\|_{\Gamma_{\gamma}^{\rho}} \leq\|v\|_{\Gamma_{\gamma+\delta}^{\rho}}$, and $\|v\|_{\Gamma_{\gamma}^{\rho+1}} \leq \delta^{-1}\|v\|_{\Gamma_{\gamma+\delta}^{\rho}}$ for any $\delta, \gamma, \rho>0$. Then iteratively (losing a fraction of the original $\epsilon$ at every step) we are able to demonstrate the argument.

\section{References}

[1] Albeverio, S. and Cruzeiro, A.-B. Global flows with invariant (Gibbs) measures for Euler and Navier-Stokes two-dimensional fluids, Comm. Math. Phys. 129, No. 3, 431-444, [1990].

[2] Ambrosio, L. and Figalli A. Of flows associated to Sobolev vector fields in Wiener spaces: An approach à la DiPerna-Lions, J. Functional Analysis, 256, 179-214, [2009].

[3] Bejenaru, I., Ionescu, A. and Kenig, C. Global existence and uniqueness of Schrdinger maps in dimensions $d \geq 4$. Adv. Math. 215, no. 1, 263-291, [2007].

[4] Bejenaru, I., Ionescu, A., Kenig, C. and Tataru, D. Global Schrödinger maps in dimensions d $\geq 2$ : small data in the critical Sobolev spaces, Ann. of Math. (2) 173, no. 3, 144-1506, [2011].

[5] Bényi, A., Oh, T. and Pocovnicu, O. On the probabilistic Cauchy theory of the cubic nonlinear Schrödinger equation on $\mathbb{R}^{d}, d \geq 3$, Trans. Amer. Math. Soc. Ser. B 2, 1-50, [2015].

\footnotetext{
${ }^{14}$ i.e. for each fixed path $t \mapsto W_{t}(\omega)$ we have a unique solution.
} 
[6] Bényi, A., Oh, T., and Pocovnicu, O. Wiener randomization on unbounded domains and an application to almost sure well-posedness of NLS, Excursions in Harmonic Analysis. Vol. 4, Appl. Numer. Harmon. Anal., Birkhäuser/Springer, pp. 3-25, [2015].

[7] Bogachev, V.I. Gaussian measures, Mathematical Surveys and Monographs, 62 American Mathematical Society, Providence, RI, [1998].

[8] Bourgain, J. Periodic nonlinear Schrödinger equation and invariant measures, Commun. Math. Phys. 166 pp. 1-26, [1994].

[9] Bourgain, J. Invariant measures for the 2D-defocusing nonlinear Schrödinger equation, Comm. Math. Phys. 176, no. 2, pp. 421-445, [1996].

[10] Buckdahn, R. Anticipative Girsanov transformations, Probab. Th. Rel. Fields 89, 211-238, [1991].

[11] Buckmaster, T., Nahmod, A., Staffilani, G. and Widmayer K. The surface quasi-geostrophic equation with random diffusion, Preprint arXiv:1806.03734 [math.AP], [2018].

[12] Burq, N., Thomann, L. and Tzvetkov, N. Long time dynamics for the one dimensional non linear Schrödinger equation. Ann. Inst. Fourier (Grenoble) 63, no. 6, 2137-2198, [2013].

[13] Burq, N., Thomann, L., and Tzvetkov, N. Remarks on the Gibbs measures for nonlinear dispersive equations, arXiv:1412.7499, [2014].

[14] Burq, N. and Tzvetkov, N. Random data Cauchy theory for supercritical wave equations. I. Local theory. Invent. Math. 173, no. 3, 449-475, [2008].

[15] Burq, N. and Tzvetkov, N. Random data Cauchy theory for supercritical wave equations. II. A global existence result. Invent. Math. 173, no. 3, 477-496, [2008].

[16] Cambronero, S. H. and McKean, H. The ground state eigenvalue of Hills equation with white noise potential, Comm. Pure and Applied Mathematics LII, 1277-1294, [1999].

[17] Cameron, R. H. and Martin, W. T. Transformation of Wiener integrals under translations, Annals of Math. 45 no.2, 386-396, [1944].

[18] Colliander, J., Keel, M., Staffilani, G., Takaoka, H. and Tao, T. Global well-posedness and scattering for the energy-critical nonlinear Schrödinger equation in $\mathbb{R}^{3}$, Ann. of Math. (2) 167, no. 3, 767-865, [2008].

[19] Colliander, J. and Oh, T. Almost sure well-posedness of the cubic nonlinear Schrödinger equation below $L^{2}(\mathbb{T})$, Duke Math. J. 161, no. 3, 367-414, [2012].

[20] Constantin, P. and Foias, C. Navier-Stokes Equations, Chicago Lectures in Mathematics, The University of Chicago Press, [1988].

[21] Cote, R., Kenig, C., Lawrie, A. and Schlag, W. Characterization of large energy solutions of the equivariant wave map problem: II, Amer. J. Math. 137, no. 1, 209-250, [2015].

[22] Cote, R., Kenig, C., Lawrie, A. and Schlag, W. Characterization of large energy solutions of the equivariant wave map problem: I, Amer. J. Math. 137, no. 1, 139-207, [2015].

[23] Cote, R., Kenig, C. and Merle, F. Scattering below critical energy for the radial 4 D Yang-Mills equation and for the 2D corotational wave map system, Comm. Math. Phys. 284, no. 1, 203-225, [2008].

[24] Deng, Y. Two-dimensional nonlinear Schrödinger equation with random radial data, Anal. PDE 5 no. 5 , 913-960, [2012].

[25] Dodson, B., Lührmann, J. and Mendelson, D. Almost sure local well-posedness and scattering for the $4 D$ cubic nonlinear Schrödinger equation, Preprint arXiv:1802.03795.

[26] Duyckaerts, T., Kenig, C. and Merle, F. Universality of blow-up profile for small radial type II blow-up solutions of the energy-critical wave equation, J. Eur. Math. Soc. (JEMS) 13, no. 3, 533-599, [2011].

[27] Duyckaerts, T., Kenig, C. and Merle, F. Profiles of bounded radial solutions of the focusing, energy-critical wave equation, Geom. Funct. Anal. 22, no. 3, 639-698, [2012].

[28] Duyckaerts, T., Kenig, C. and Merle, F. Universality of the blow-up profile for small type II blow-up solutions of the energy-critical wave equation: the nonradial case, J. Eur. Math. Soc. (JEMS) 14, no. 5, 1389-1454, [2012].

[29] Enchev, O. Nonlinear Transformations on the Wiener Space, The Annals of Probability, 21, no 4, 21692188 [1993].

[30] Flandoli, F. Random perturbation of PDEs and fluid dynamic models. 2015, Lecture Notes in Mathematics. Springer, Heidelberg, 2011. Lectures from the 40th Probability Summer School held in Saint-Flour, École d'Été de Probabilités de Saint-Flour, [2010].

[31] Foias, C. and Temam R. Gevrey class regularity for the solutions of the Navier-Stokes equations, J. Funct. 
Anal., 87 no. 2 359-369, [1989].

[32] Glatt-Holtz, N. and Vicol, V. Local and global existence of smooth solutions for the stochastic Euler equations with multiplicative noise, Ann. Probab., 42 no. 1, 80-145, [2014].

[33] Grünrock, A. and Herr, S. Low regularity local well-posedness of the derivative nonlinear Schrödinger equation with periodic initial data, SIAM J. Math. Anal. 39, 1890-1920, [2008].

[34] Herr, S., Tataru, D. and Tzvetkov, N. Global well-posedness of the energy-critical nonlinear Schrödinger equation with small initial data in $H^{1}\left(\mathbb{T}^{3}\right)$, Duke Math. J., 159 329-349, [2011].

[35] Ionescu, A. D. and Pausader, B. The energy-critical defocusing NLS on $\mathbb{T}^{3}$, Duke Math. J. 161 no. 8 , 1581-1612, [2012].

[36] Kallianpur G. and Karandikar, R. L. Nonlinear Transformations of the canonical Gauss measure on Hilbert space and absolute continuity, Acta Applicandae Mathematicae 35, 63-102, [1994].

[37] Kenig, C. Oscillatory integrals and nonlinear dispersive equations. Essays on Fourier analysis in honor of Elias M. Stein (Princeton, NJ, 1991), 268-285, Princeton Math. Ser., 42, Princeton Univ. Press, Princeton, NJ, [1995].

[38] Kenig, C. The concentration-compactness rigidity method for critical dispersive and wave equations, Nonlinear partial differential equations, 117-149, Adv. Courses Math. CRM Barcelona, Birkhuser/Springer Basel AG, Basel, [2012].

[39] Kenig, C. and Merle, F. Global well-posedness, scattering and blow-up for the energy-critical, focusing, non-linear Schrödinger equation in the radial case, Invent. Math. 166, no. 3, 645-675, [2006].

[40] Kenig, C. and Merle, F. Global well-posedness, scattering and blow-up for the energy-critical focusing non-linear wave equation, Acta Math. 201, no. 2, 147-212, [2008].

[41] Kenig, C. and Merle, F. Scattering for $H^{1} / 2$ bounded solutions to the cubic, defocusing NLS in 3 dimensions, Trans. Amer. Math. Soc. 362, no. 4, 1937-1962, [2010].

[42] Kenig, C., Ponce, G. Rolvung, C. and Vega, L. Variable coefficient Schrdinger flows for ultrahyperbolic operators, Adv. Math. 196, no. 2, 373-486, [2005].

[43] Kenig, C., Ponce, G. and Vega, L. Well-posedness and scattering results for the generalized Korteweg-de Vries equation via the contraction principle, Comm. Pure Appl. Math. 46, no. 4, 527-620, [1993].

[44] Kenig, C., Ponce, G. and Vega, L. The Cauchy problem for the Korteweg-de Vries equation in Sobolev spaces of negative indices, Duke Math. J. 71, no. 1, 1-21, [1993].

[45] Kenig, C., Ponce, G. and Vega, L. Quadratic forms for the 1D semilinear Schrödinger equation, Transaction of the AMS, 348, 3323-3353, [1996].

[46] Kenig, C., Ponce, G. and Vega, L. A bilinear estimate with applications to the KdV equation, J. Amer. Math. Soc. 9, no. 2, 573-603, [1996].

[47] Kenig, C., Ponce, G. and Vega, L. Smoothing effects and local existence theory for the generalized nonlinear Schrödinger equations, Invent. Math. 134, no. 3, 489-545, [1998].

[48] Kenig, C., Ponce, G. and Vega, L. On the concentration of blow up solutions for the generalized KdV equation critical in $L^{2}$, Nonlinear wave equations (Providence, RI, 1998), 131-156, Contemp. Math., 263, Amer. Math. Soc., Providence, RI, [2000].

[49] Kenig, C., Ponce, G., and Vega, L. On the ill-posedness of some canonical dispersive equations, Duke Math. J. 106, no. 3, 617-633, [2001].

[50] Kenig, C., Ponce, G. and Vega, L. The Cauchy problem for quasi-linear Schrödinger equations, Invent. Math. 158, no. 2, 343-388, [2004].

[51] Kusuoka, S. The nonlinear transformation of Gaussian measure on Banach space and its absolute continuity (I), J. Fac. Univ. Tokyo Sect. IA Math. 29, no. 3, 567-597, [1982].

[52] Lührmann, J. and Mendelson, D. Random data Cauchy theory for nonlinear wave equations of power-type on $\mathbb{R}^{3}$, Comm. Partial Differential Equations 39, no. 12, 2262-2283, [2014].

[53] Lührmann, J. and Mendelson, D. On the almost sure global well-posedness of energy sub-critical nonlinear wave equations on $\mathbb{R}^{3}$, New York J. Math. 22, 209-227, [2016].

[54] Nahmod, A. R., Oh, T., Rey-Bellet, L. and Staffilani, G. Invariant weighted Wiener measures and almost sure global well-posedness for the periodic derivative NLS, J. Eur. Math. Soc. (JEMS) 14, no. 4, pp. 12751330, [2012].

[55] Nahmod, A. R., Pavlovic, N., and Staffilani, G. Almost sure existence of global weak solutions for supercritical Navier-Stokes equations, SIAM J. Math. Anal. 45, no. 6, 3431-3452, [2013]. 
[56] Nahmod, A. R., Pavlovic, N., Staffilani, G., and Totz, N., Global Flows with Invariant Measures for the Inviscid Modified SQG Equations, Stoch. Partial Differ. Equ. Anal. Comput. 6, no. 2, 184-210, [2018].

[57] Nahmod, A. R., Rey-Bellet, L., Sheffield, S. and Staffilani, G. Absolute continuity of Brownian bridges under certain gauge transformations, Math. Res. Lett. 18, no. 5, pp. 875-887, [2011].

[58] Nahmod, A. R. and Staffilani, G. Almost sure well-posedness for the periodic 3D quintic nonlinear Schrödinger equation below the energy space, JEMS, 17, no. 7, 1687-1759, [2015].

[59] Oh, T. and Thomann, L., A pedestrian approach to the invariant Gibbs measures for the 2-d de-focusing nonlinear Schrödinger equations, Stoch. Partial Differ. Equ. Anal. Comput, [2018].

[60] Oksendal, B. Stochastic Differential Equations, Springer-Verlag, [2003].

[61] Quastel, J. and Valkó, B. KdV Preserves White Noise, Communications in Mathematical Physics 277, no. 3, 707-714, [2008].

[62] Ramer, R. On nonlinear transformations of Gaussian measures, J. Functional Anal. 15, 166-187, [1974].

[63] Thomann, L. and Tzvetkov, N., Gibbs measure for the periodic derivative nonlinear Schrödinger equation, Nonlinearity 23, no. 11, 2771-2791, [2010]. 